\title{
DESIGNING A DISRUPTION-AWARE SUPPLY CHAIN NETWORK CONSIDERING PRECAUTIONARY AND CONTINGENCY STRATEGIES: A REAL-LIFE CASE STUDY
}

\author{
Mohammad Hossein Dehghani Sadrabadi@, \\ Rouzbeh Ghousi*® and Ahmad Makui®
}

\begin{abstract}
Due to the high risk in the business environment, supply chains must adopt a tailored mechanism to deal with disruptions. This research proposes a multi-objective formulation to design a robust and resilient forward supply chain under multiple disruptions and uncertainty. The mentioned objective functions include minimizing the total cost, environmental impacts, and the network nonresiliency associated with the supply chain simultaneously countered using an augmented $\varepsilon$-constraint method. A Mulvey robust optimization approach is also utilized to deal with uncertainty. Ultimately, the developed model is validated based on three datasets associated with a case study of the steel industry. The results indicate that preventive and mitigation resilience strategies have significantly promoted the supply chain's capabilities to deal with disruptions. Controlling network resiliency via non-resiliency measures has also created a risk-aware and robust structure in the incidence of disturbances. Numerical results reveal that multiple sourcing, lateral transshipment, and fortification of facilities will lead to the greatest cost-efficiency in the case study. Observations also indicate that the fortified supply chain will be highly economically viable in the long run due to the reduction of costs resulting from lost sales, unnecessary inventory holding, and the company's credit risk.
\end{abstract}

Mathematics Subject Classification. 90B06.

Received April 6, 2021. Accepted August 11, 2021.

\section{INTRODUCTION}

A supply chain (SC) is a network comprising various facilities located and organized for significant purposes. The coordination of operations in SC can reduce costs, improve the service level, and promote customer satisfaction $[16,51]$. SC management (SCM) precisely plans resources and limitations to locate facilities and allocate the flow of materials and products between them to achieve systematically desired goals $[27,36]$. An appropriate design has a significant impact on performance, profitability, and customer satisfaction in SC. Making wise decisions in SC has a lasting effect on both strategic and operational goals [10,20].

Due to the turbulent business environment, SCs may face different threats and risks that can have lasting impacts on system performance. Zhalechian et al. [49] considered SC threats to be disruptive and operational in resilient SC network design (RESCND) problems. Note that operational risks and disturbances have entirely

Keywords. Resilient system; network non-resiliency; robust optimization; disruption; operational risk.

Department of Industrial Engineering, Iran University of Science \& Technology, Tehran, Iran.

*Corresponding author: ghousi@iust.ac.ir 
different natures. Given the destructive effects of risks on the SC, a tailored mechanism should be applied to cope with such problems and improve system performance and reliability $[41,50]$.

Businesses have to cautiously prepare for disruptions and operational fluctuations to prevail in risks. In the absence of appropriate and applicable planning for operational risk and disruption management, the recovery of a disrupted SC requires considerable time and costs, as well as tolerating severe damages, such that the performance of the SC is completely disrupted and cannot be recovered [33,37]. Therefore, the SC network design (SCND) problem must be implemented such that it can resiliently and responsibly withstand all operational risks and disturbances, including long and short-term fluctuations and hazards. In the literature, resiliency is commonly defined as the system's ability to recover normal performance or achieve a more eligible state after disruption $[32,38]$. SC resilience (SCRES) consists of some mitigation and preventive strategies to counter disruptions that develop a resilient system in the face of disturbances and operational risks. Mitigation or contingency measures are executed after disruption to pay for damages and restore desired performance or a better state $[34,35]$. Preventive measures are those strategies considered by organizations before the incidence of disruption to prepare for avoiding losses and failures [4].

Accordingly, resilience is a procedure for SC to prevent or reduce the damages caused by disruptions. The mentioned mechanism can restore the system's regular performance or achieve a better state [11]. Resilience enables companies to manage SC failures and continue delivering their products and services to customers [40]. RESCND tackles possible disruptions and is an essential procedure in SCM. SCRES consists of various strategies to mitigate the detrimental influences of disturbances on SC. SCM must adopt protective measures to prevent system failure [22]. Consequently, RESCND has recently become an attractive field of research.

The present study addresses a resilient and robust forward SCND problem in the incidence of multiple disruptions and uncertainty. The proposed formulation is multi-product, multi-echelon, two-stage, and multiperiod to cover research gaps and enrich the literature. Moreover, the considered objective functions (OFs) include minimizing the total cost (TC), the total environmental impact (TEI), and the total non-resiliency of the network (TNRN) associated with the SC. Herein, mitigation and precautionary resilience measures, including holding pre-positioned emergency inventory (HPEI), multiple sourcing (MS), taking advantage of lateral transshipment (LT), fortification of facilities (FF), and providing backup facilities (PBF), are considered simultaneously. Besides, non-resiliency measures such as NC, FC, and NCr are employed to develop an SC with a resilient network. The facilities are assumed to be partially disrupted, meaning that any disturbance can only damage a fraction of their capacity. On the other hand, it is assumed that routes or transportation links are completely disrupted so that a disrupted route will not be available. Furthermore, multiple disturbance is addressed by considering simultaneous disruption in facilities, the routes between them, and transshipment links. Notably, we simultaneously employed structural resilience strategies and measures of network non-resiliency to develop a network-resilient SC and cope with disruptions.

Some decisions are made by solving the suggested mathematical model. The main decisions include locating warehouses, production centers, and distribution centers; selecting proper backup suppliers; specifying the flow between facilities; and determining the number of lost sales. Besides, investigating the FF at a particular level under risks of disturbance, specifying the amount of pre-positioned EI that must be kept at fortified facilities, and specifying the amount of purchase from the pre-positioned EI of fortified facilities are extracted as resilience decisions.

This paper is organized as follows: Section 2 reviews the relevant studies on the RESCND pertaining to operational and disruption risk. In Section 3, the problem is defined, and the mathematical formulation is proposed. Section 4 describes the transformation of the deterministic multi-objective model into a robust stochastic single-objective one. In Section 5, the model is solved based on a real-world case problem, and the results are extracted. Finally, conclusions and avenues for further research are discussed in Section 6. 


\section{Literature REVIEW}

SCs face various risks throughout their lives, including operational risks and disruptions that can have detrimental impacts on performance and profitability. Disruptions or risks of disturbance are devastating disasters such as human faults, terrorist attacks, economic corruption, and natural disasters [28, 43]. Moreover, the usual fluctuations and difficulties in the business environment that lead to inherent uncertainties in the SCND problems are known as operational risks. This type of SC threat is presented in four categories: supplier, environmental, internal, and market risks, which can have environmental or systematic origins [44]. Supplier risks involve contractual threats with suppliers, indeterminacy in transmission, abuse of profits with suppliers, and a lack of flexibility in the supply process and lead time. Environmental risks arise from fluctuating energy costs, social issues, globalization rules, governments, regulatory laws, competitors' initiatives, emerging technology, joint-venture policies, and the damaging impact of media. Furthermore, internal risks include indeterminacy in credit and labor, unacceptable staffing, improper productivity, policy fluctuations, alternations in processes, organizational managers' replacement, investment challenges, and insufficient operational knowledge. Finally, market risks arise from limited insight into market turmoil, customers' needs, improper products, and aftersales service; changes in customers' preferences; providing alternative products or services; delays in meeting demands; the inability of demand anticipation and estimation; a lack of agility in meeting demands; and the like $[9,45]$.

SCs must always be prepared to deal with risks ahead and take appropriate action. An organization's inability to fulfill customer demand has severe and irreversible consequences such as increasing delivery time, reducing brand credibility, losing customer trust and satisfaction, and price inflation. SCRES helps empower the network to respond to sudden risks by preventing, mitigating, or transmitting damages [8]. Although different definitions have been suggested for resiliency, it is commonly defined as the system's ability to recover typical performance or achieve a more eligible state after disruption [32]. Studies on RESCND focus on different precautionary and contingency resilience strategies to prevent or reduce the devastating impacts of various disruptions on SC. The primary and common resilience strategies include the FF, network resiliency, MS, HPEI, PBF, scenario-based disruption, and maintaining safety stock.

Many researchers have studied the concept of resilience, mitigation plans, contingency strategies, and implementation in the SC. For instance, Sawik [39] proposed a model for supply portfolio selection under disturbance risk. This study sought to activate suppliers and allocate products in a disrupted business environment. Silbermayr and Minner [42] discussed dual sourcing and cost-saving under disruption risks through organizational learning. This study examined a balance between risk mitigation by utilizing multiple suppliers in the disruption situation and the benefits of learning over-sourcing costs to create a decision support system. Rezapour et al. [35] proposed RESCND in a competitive environment. In their research, facilities were under partial disturbance, and they implemented several resilience strategies. Fattahi et al. [12] proposed a RESCND under operational risks and disruption consideration with lead time sensitivity and applied some mitigation and preventive resilience strategies simultaneously. Mohammed et al. [24] presented RESCND while taking into account the disruption risk. They developed a green and resilient model in a fuzzy environment to achieve strategic and operational aspirations, and tried to minimize the TC and TEI, and maximize resiliency pillars value. Pavlov et al. [31] addressed a RESCND while considering sustainability dimensions under disturbance risks. They employed redundancy and mitigation approaches to reduce the impacts of disruptions. Besides, coordination and resiliency were simultaneously applied in the proposed mathematical model. Garcia-Herreros et al. [13] discussed RESCND under disturbance risks, and took into account complete facility disruption. They also applied two-stage stochastic programming (TSSP) to cope with possible risks using disruption scenarios. Azad et al. [1] proposed a RESCND model to disrupt distribution centers and transportation links. They provided backup facilities and applied FF to diminish the destructive influences of disruptions. Nooraie and Parast [29] discussed SCRES under partial facilities disruption and proposed some contingency resiliency measures such as MS and PBF. Ghavamifar et al. [14] developed a resilient SC under disruptions in a competitive environment. They considered complete facility disturbance and some resilience measures. Zahiri et al. [48] proposed a resilient and 
sustainable pharmaceutical SC in the incidence of disturbances and operational risks, and applied a robust optimization (RO) method to deal with operational risks. Sabouhi and Jabalameli [36] discussed RESCND under disruptions, and utilized various strategies to minimize the TC of the system and the TNRN. Hosseini-Motlagh et al. [18] developed a resilient SC considering minimum TNRN, TC, and maximum social welfare.

A vast majority of studies on SCRES employed contingency and precautionary measures for RESCND under various disruptions. Notably, some of these studies attempted to minimize the TNRN and the TC of the system. TNRN is a new field of research in the area of RESCND and has several components. Based on the reviewed studies, node complexity $(\mathrm{NC})$, flow criticality ( $\mathrm{FCr}$ ), network density (ND), node criticality ( $\mathrm{NCr}$ ), and flow complexity (FC) are measures of the TNRN. Note that $[5,18,36,48]$ are the only studies mentioning an improvement in the resiliency of SC by reducing the TNRN.

A number of research gaps were identified. First, the reviewed studies did not adequately discuss multiechelon RESCND in the case of disruptions. Second, contingency and precautionary resilience measures have rarely been applied simultaneously in the field of RESCND, and the vast majority of studies merely took into account a particular type of strategy. Third, a few relevant studies have discussed multiple disruptions, including simultaneous or sequential disturbance in facilities and transportation links. Fourth, LT has not received sufficient attention as a resilience measure in the case of disturbance. Fifth, uncertainty and disruptions have scarcely been considered simultaneously. Sixth, network non-resiliency measures such as NC, NCr, FC, FCr, and ND have not been widely discussed in the domain of RESCND.

Given the identified research gaps, this paper aims to fortify the literature on RESCND in several different directions. Based on the reviewed studies, this paper proposes a multi-objective multi-period robust and RESCND model to minimize the TC, TEI, and TNRN simultaneously. The mentioned SC is entangled with multiple disruptions of facilities, the routes between them, and related links. Moreover, the usual uncertainty due to the inherent risk of parameters and business environment instability is considered. TSSP is employed to apply the disruption scenarios in the proposed model. Ultimately, a robust stochastic programming approach is applied to counter both operational and disruption risks simultaneously.

\section{A HYBRID APPROACH FOR RESCND}

\subsection{Problem description}

In this paper, a resilient and robust forward SC under operational risk and multiple disturbances is developed. The proposed formulation is multi-product, multi-echelon, two-stage, multi-period, and risk-vulnerable. The configuration of this forward SC is as follows.

Based on Figure 1, the concerned SC consists of six echelons, including primary and backup suppliers, warehouses, production centers, distribution centers, and first markets. The concerned SC involves forward flow so that warehouses receive raw materials from both primary and backup suppliers for storage. After that, production centers receive raw materials from warehouses to manufacture products. Ultimately, distribution centers receive products from production centers to deliver them to the applicant customers in the primary market. The relevant network includes supplying and storing raw materials, manufacturing products using raw materials, and finally, sending them to retailers and wholesalers for distribution. The strategic end products include steel alloys widely used in numerous industries, especially parent industries. Steel is an iron alloy with a carbon content of $0.002-2.1 \%$ by weight. Its properties can be controlled by altering the percentage of carbon, alloying elements, and heat treatment. The most important alloying elements in steel include carbon, manganese, silicon, phosphorus, sulfur, chromium, nickel, molybdenum, cobalt, tungsten, vanadium, titanium, niobium, aluminum, tin, copper, lead, nitrogen, and hydrogen. However, the added percentage of tin, copper, lead, nitrogen, and hydrogen in steel production is small. Owing to its high tensile strength and relatively low cost, steel is widely utilized in various industries such as construction, infrastructure, tools, ships, trains, automobiles, machinery, equipment, and military weapons.

In general, steel alloys are produced via two technologies. The first method involves using electric arc furnaces to melt sponge iron and produce the alloy. The second method uses crude iron (iron produced in the indirect 


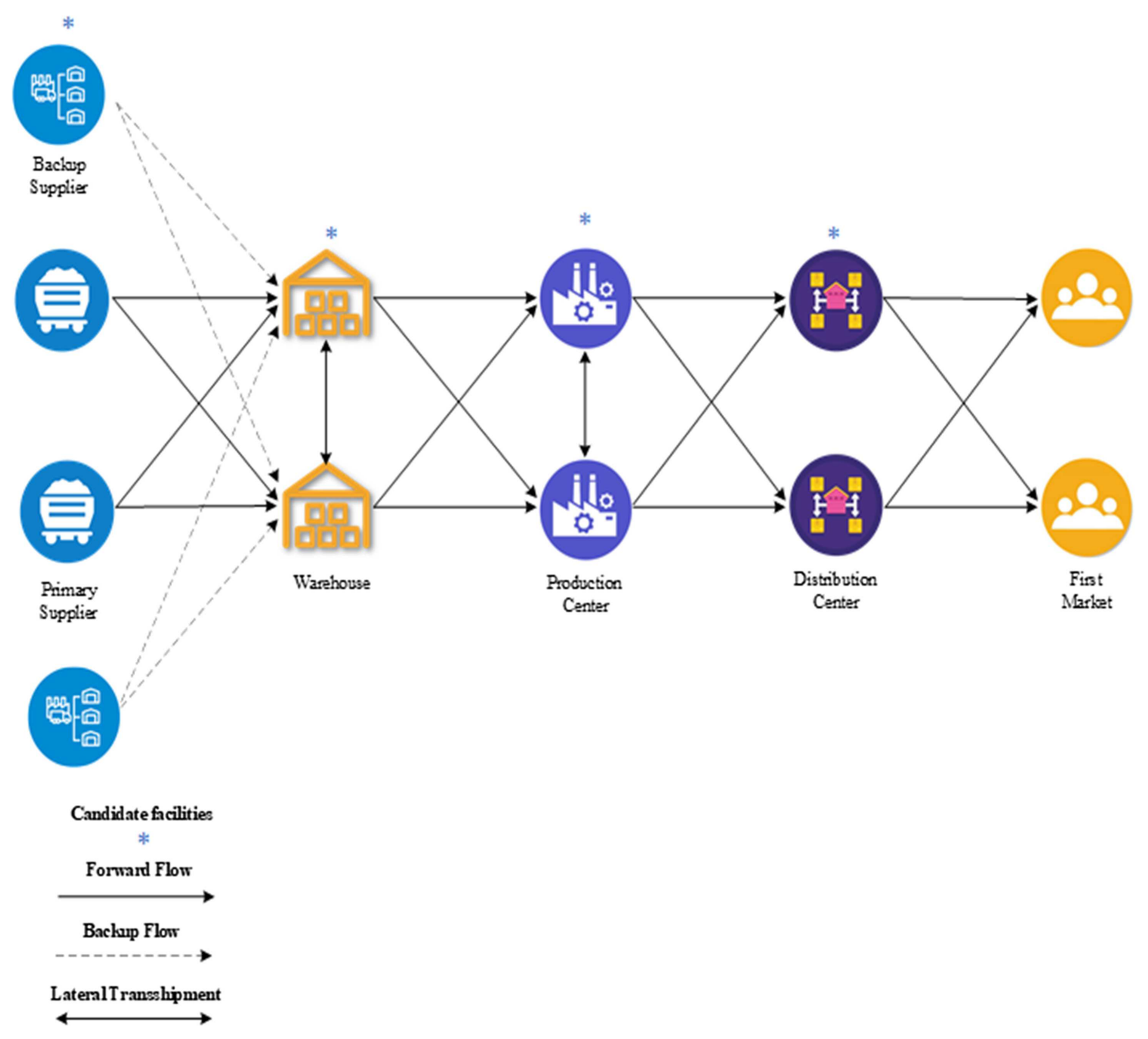

Figure 1. The configuration of considered forward supply chain.

reduction process) and burns the excess carbon [46]. During this process, the amount of crude iron-carbon is reduced from 5.3 to $6,2.0$, and $1.5 \%$ by weight; then, other elements are added to obtain the desired composition. Iron is commonly found in minerals such as magnetite and hematite in the Earth's crust. Steel is produced by pulverizing and smelting it by burning the carbon of white crude iron, melting it with a slight amount of carbon, and measuring other elements. Steel containing up to $0.2 \%$ carbon is used to make steel wires, pipes, and sheets. Besides, steel with an average of $0.2-0.6 \%$ carbon is utilized to make rails, boilers, and building components. Ultimately, the alloy with $0.6-1.5 \%$ carbon is hard and is used to make tools, springs, and cutlery [47]. The products of steel companies include various types of ingots, iron sheets, rebars, iron angles, pipes, and beams widely employed in multiple industries. The case study in the present research is based on the steel industry in Iran. We implemented the case study in the proposed framework to ensure the applicability of the proposed formulation.

There are essential points in SCND for the steel industry, some of which are described below. The quality of raw materials strongly affects the properties of steel products and their applicability in various industries. Accordingly, the delivery of poor-quality raw materials or failure to comply with the prescribed lead time by a 
primary supplier causes the defective supplier to be immediately substituted with a backup supplier. The backup supplier undertakes to provide raw materials with sufficient quality at the time committed for the production process in case of disruptions. In addition, any disruption in the production process of steel alloys leads to delays in orders of parent industries and dissatisfaction of customers, so fortifying the infrastructure of the facilities is crucial in SCND for the steel industry. According to the above explanations, some measures must be taken to deal with disruptions in the steel SC, described below.

This study considers multiple disturbances in facilities and transportation links in the proposed SC where facilities and routes are partially and wholly disrupted, respectively. Note that the situation resulting from the occurrence of each disruption is wisely demonstrated by defining a set of independent scenarios. Besides, the impacts of scenarios are tackled by applying TSSP that requires two-phase decision-making, including strategic (pre-event) and operational decisions (post-event). The pre-event or first stage includes scenario-independent decisions, while the post-event or second stage deals with scenario-dependent decisions. Strategic decisions associated with the model include locating warehouses, production centers, and distribution centers; selecting backup suppliers; and determining the facilities' fortification level. Besides, specifying the amount of product transported among facilities, the amount of pre-positioned EI that should be kept, the level of typical inventory at some facilities, and the amount of lost sale in first markets are the as operational decisions.

This study investigates both network and structural resilience strategies to mitigate the destructive consequences of disturbances. The considered mitigation and precautionary resilience measures include holding pre-positioned EI, MS, taking advantage of LT, FF, providing backup suppliers, and scenario-based disruption. Besides, NCr, NC, and FC are considered as the TNRN measures to develop a network-resilient SC. Note that the usual operational risks associated with the instability of the business environment and inherent uncertainties in parameters are tackled by applying a robust stochastic programming model.

This research investigates a robust stochastic multi-objective multi-echelon model for the RESCND problem to attain optimal strategic and operational decisions. The OFs include minimizing the TC, the TEI of SC, and the TNRN. Besides, the issue of multiple OFs is countered by employing an augmented $\varepsilon$-constraint method.

Further assumptions associated with the model are as follows:

- The candidate locations for establishing new facilities are known.

- Only a single route can be opened between two facilities.

- Facilities and routes are partially and completely disrupted, respectively.

- Multiple disturbances are considered in facilities and transportation links.

- Disturbance scenarios are considered independently and with a predefined probability.

- Facilities can serve to their maximum capacity.

- In case of a shortage in markets, it is considered a lost sale.

- Backup facilities are not disrupted in the incidence of disturbance.

- All of the routes between backup suppliers and warehouses can be utilized for material transmission.

Herein, a hybrid approach is deployed to make strategic and operational decisions associated with the SC in the event of disruptions and operational risks. This framework entails developing a robust multi-objective formulation for the RESCND problem in the case of uncertainty and disturbances. Accordingly, the obtained technical, economic, environmental, resiliency parameters, facilities with satisfactory efficiency, and the corresponding coefficients are incorporated as primary input parameters of the robust RESCND model. The problem is subjected to some constraints, including flow balance, inventory balance, resiliency consideration, demand fulfillment, allocations conditions, disrupted route selection, and process or operational considerations. Besides, the proposed model aims to minimize the expected TC, TEI, and TNRN simultaneously. The presented formulation is entangled with some issues, including multiple OFs and the incidence of operational risks or uncertainty. Due to these issues, the multi-objective model is converted into a single-objective formulation by employing an augmented $\varepsilon$-constraint method. Furthermore, operational risks are coped with by developing a scenario-based robust (SBR) formulation based on Mulvey's RO technique in the generated equivalent formulation. Eventually, the model is coded in the optimization software and solved by taking advantage of an appropriate solver. 
Eventually, the managerial and numerical results are extracted, and all strategic and operational decisions are made. Strategic or pre-event decisions include the optimal location of facilities involved in the SC, determining fortification levels for fortified facilities, selecting backup suppliers, and identifying critical nodes in the SC. On the other hand, operational or post-event decisions entail the optimal flow between network nodes, the level of inventory held at facilities, allocation of facilities to each other, the level of pre-positioned EI at fortified facilities, and the number of products or materials purchased from the EI of fortified facilities. In this stage, sensitivity analysis is also implemented to assess the impact of parameters associated with SCND and robustness on OFs and evaluate the conflicts between them. Ultimately, the outcomes of the proposed robust and resilient formulation and model validation are precisely discussed.

\subsection{Model formulation}

The concerned SC is formulated from the perspective of the involved stakeholder. The indices, parameters, and decision variables in the mathematical formulation are introduced below. The concerned SC is modeled according to TSSP [17], and the corresponding two-stage formulation is presented in what follows.

\section{Sets}

$C \quad$ Index of products first market zones $\quad c \in C$

$D \quad$ Index of potential locations for distribution centers $\quad d \in D$

$I \quad$ Index of raw material families

$M \quad$ Index of potential locations for production centers $\quad m, m^{\prime} \in M$

$L \quad$ Index of fortification levels $\quad l \in L$

$P \quad$ Index of products families $\quad p \in P$

$S \quad$ Index of potential locations primary suppliers $\quad s \in S$

$B \quad$ Index of available backup suppliers $\quad b \in B$

$W \quad$ Index of potential locations for warehouses $\quad w, w^{\prime} \in W$

$R$ Index of available routes $\quad r \in R$

$V \quad$ Index of production technologies $\quad v \in V$

$O$ Index of disruptions $\quad o \in O$

$T$ Index of periods $\quad o \in T$

\section{Parameters}

\begin{tabular}{|c|c|}
\hline $\mathrm{FOD}_{d}$ & Opening cost of distribution center $d$ (million rials) \\
\hline $\mathrm{FOM}_{m}$ & Opening cost of production center $m$ (million rials) \\
\hline $\mathrm{FOW}_{w}$ & Opening cost of warehouse $w$ (million rials) \\
\hline $\mathrm{FFM}_{m l}^{\infty}$ & Fortification cost of production center $m$ at level $l$ (million rials) \\
\hline $\mathrm{FFS}_{s l}$ & Fortification cost of the primary supplier $s$ at level $l$ (million rials) \\
\hline $\mathrm{FCB}_{b}$ & Cost of contracting with backup supplier $b$ (million rials) \\
\hline $\mathrm{TMD}_{p m d r t}^{o}$ & $\begin{array}{l}\text { Unit transportation cost of the } p \text {-type product from production center } m \text { to distribution center } \\
d \text { by route } r \text { in period } t \text { in the incidence of disturbance } o \text { (million rials/ton.km) }\end{array}$ \\
\hline $\mathrm{TMM}_{p m m^{\prime} r t}^{o}$ & $\begin{array}{l}\text { Unit lateral transportation cost of the } p \text {-type product from production center } m \text { to production } \\
\text { center } m^{\prime} \text { by route } r \text { in period } t \text { in the incidence of disturbance } o \text { (million rials/ton.km) }\end{array}$ \\
\hline $\mathrm{TWM}_{i w m r t}^{o}$ & $\begin{array}{l}\text { Unit transportation cost of } i \text {-type raw material from warehouse } w \text { to production center } m \text { by } \\
\text { route } r \text { in period } t \text { in the incidence of disturbance } o \text { (million rials } / \text { ton.km) }\end{array}$ \\
\hline $\mathrm{TWW}_{i w w^{\prime} r t}^{o}$ & $\begin{array}{l}\text { Unit lateral transportation cost of } i \text {-type raw material from warehouse } w \text { to warehouse } w^{\prime} \text { by } \\
\text { route } r \text { in period } t \text { in the incidence of disturbance } o \text { (million rials/ton.km) }\end{array}$ \\
\hline $\mathrm{TSW}_{\text {iswrt }}^{o}$ & $\begin{array}{l}\text { Unit cost of purchasing } i \text {-type raw material from primary supplier } s \text { and transporting to } \\
\text { warehouse } w \text { by route } r \text { in period } t \text { in the incidence of disturbance } o \text { (million rials/ton.km) }\end{array}$ \\
\hline $\mathrm{TBW}_{\text {ibwrt }}^{o}$ & $\begin{array}{l}\text { Unit cost of purchasing } i \text {-type raw material from backup supplier } b \text { and transporting to ware- } \\
\text { house } w \text { by route } r \text { in period } t \text { in the incidence of disturbance } o \text { (million rials/ton.km) }\end{array}$ \\
\hline
\end{tabular}


$\mathrm{HCMI}_{i p m t}^{o} \quad$ Unit cost of holding $i$-type raw material of $p$-type product at production center $m$ in period $t$ in the incidence of disturbance $o$ (million rials/ton)

$\mathrm{HCW}_{i w t}^{o} \quad$ Unit cost of holding $i$-type raw material at warehouse $w$ in period $t$ in the incidence of disturbance $o$ (million rials/ton)

$\mathrm{PC}_{p m v t}^{o} \quad$ Unit manufacturing cost of the $p$-type product at the production center $m$ by production technology $v$ in period $t$ in the incidence of disturbance $o$ (million rials/ton)

$\mathrm{SCC}_{p c t}^{o} \quad$ Unit shortage cost of lost sales for the $p$-type product at products first market $c$ in period $t$ in the incidence of disturbance $o$ (million rials/ton)

$\mathrm{DCD}_{\text {pdcrt }}^{o} \quad$ Unit distribution cost of the $p$-type product by distribution center $d$ to products first market $c$ by route $r$ in period $t$ in the incidence of disturbance $o$ (million rials/ton.km)

$\beta_{o} \quad$ Unit penalty cost of remaining capacity of facilities in the incidence of disturbance $o$ (million rials/ton)

$\mathrm{BEMD}_{p m d t}^{o} \quad$ Unit cost of providing $p$-type product from the EI of fortified production center $m$ by distribution center $d$ in period $t$ in the incidence of disturbance $o$ (million rials/ton)

$\mathrm{BESW}_{\text {iswt }}^{o} \quad$ Unit cost of providing $i$-type raw material from the EI of the fortified primary supplier $s$ by warehouse $w$ in period $t$ in the incidence of disturbance $o$ (million rials/ton)

PIFM $_{p m t}^{o} \quad$ Unit cost of holding pre-positioned EI of $p$-type product at fortified production center $m$ in period $t$ in the incidence of disturbance $o$ (million rials/ton)

PIFS $_{i s t}^{o} \quad$ Unit cost of holding pre-positioned EI of $i$-type raw material at the fortified primary supplier $s$ in period $t$ in the incidence of disturbance $o$ (million rials/ton)

$\mu^{\prime} \quad$ Unit penalty coefficient for NC of warehouses

$\mu^{\prime \prime} \quad$ Unit penalty coefficient for NC of production centers

$\mu^{\prime \prime \prime} \quad$ Unit penalty coefficient for NC of distribution centers

$\phi \quad$ Unit penalty coefficient of FC between primary supplier $s$ and warehouse $w$

$\phi^{\prime} \quad$ Unit penalty coefficient of FC between warehouse $w$ and production center $m$

$\phi^{\prime \prime}$

$\phi^{\prime \prime \prime}$

Unit penalty coefficient of FC between production center $m$ and distribution center $d$

$\theta^{\prime}$

$\theta^{\prime \prime}$

$\theta^{\prime \prime \prime}$

$d_{\text {swr }}$

$d_{\text {bwr }}$

$d_{w m r}$

$d_{m d r}$

$d_{d c r}$

$d_{m m^{\prime} r}$

$d_{w w^{\prime} r}$

Scaps $_{s i}$

Scapwi $_{w i}$

Unit penalty coefficient of FC between distribution center $d$ and first market $c$

Unit penalty coefficient for critical warehouses

Unit penalty coefficient for critical production centers

$H_{\text {capw }}$ wi

Pcapm $_{m p v}$

Unit penalty coefficient for critical distribution centers

Distance between primary supplier $s$ and warehouse $w$ by route $r(\mathrm{Km})$

Distance between backup supplier $b$ and warehouse $w$ by route $r(\mathrm{Km})$

Distance between warehouse $w$ and production center $m$ by route $r(\mathrm{Km})$

Distance between production center $m$ and distribution center $d$ by route $r(\mathrm{Km})$

Distance between distribution center $d$ and first market $c$ by route $r(\mathrm{Km})$

Distance between production center $m$ and production center $m^{\prime}$ by route $r(\mathrm{Km})$

Distance between warehouse $w$ and warehouse $w^{\prime}$ by route $r(\mathrm{Km})$

Maximum supply capacity of primary supplier $s$ for $i$-type raw material (Ton)

Maximum supply capacity of warehouse $w$ for $i$-type raw material (Ton)

Maximum holding capacity of warehouse $w$ for $i$-type raw material (Ton)

Hcapmi $_{m i}$

$\operatorname{Dcapd}_{d p}$

$\mathrm{MHFM}_{m l p t}^{o}$

Maximum manufacturing capacity of production center $m$ for $p$-type product by production technology $v$ (Ton)

Maximum holding capacity of production center $m$ for $i$-type raw material (Ton)

Maximum delivery capacity of distribution center $d$ for $p$-type product (Ton)

$\mathrm{MHFS}_{\text {slit }}^{o}$

$p$-type product in period $t$ in the incidence of disturbance $o$ (Ton)

$S c a p b_{b i}^{o}$

Maximum holding capacity of pre-positioned EI at the fortified primary supplier $s$ at level $l$ for $i$-type raw material in period $t$ in the incidence of disturbance $o$ (Ton)

$\begin{array}{ll}S c a p b & \text { Available maximum supply capa } \\ & \text { dence of disturbance } o \text { (Ton) }\end{array}$

$\operatorname{RSS}_{\text {sit }}^{o} \quad$ Percentage of disrupted supply capacity of primary supplier $s$ for $i$-type raw material in period $t$ in the incidence of disturbance $o$ (Percentage) 
$\operatorname{RSFS}_{\text {slit }}^{o} \quad$ Percentage of disrupted supply capacity of the fortified primary supplier $s$ at level $l$ for $i$-type raw material in period $t$ in the incidence of disturbance $o$ (Percentage)

$\mathrm{RSW}_{w i t}^{o} \quad$ Percentage of disrupted supply capacity of distribution center $w$ for $i$-type raw material in period $t$ in the incidence of disturbance (Percentage)

$\mathrm{RHW}_{\text {wit }}^{o} \quad$ Percentage of the disrupted holding capacity of warehouse $w$ for $i$-type raw material in period $t$ in the incidence of disturbance $o$ (Percentage)

$\mathrm{RPM}_{m p v t}^{o} \quad$ Percentage of disrupted manufacturing capacity of production center $m$ for $p$-type product by production technology $v$ in period $t$ in the incidence of disturbance $o$ (Percentage)

$\mathrm{RPFM}_{m l p v t}^{o}$ Percentage of disrupted manufacturing capacity of fortified production center $m$ at level $l$ for $p$-type product by production technology $v$ in period $t$ in the incidence of disturbance $o$ (Percentage)

$\mathrm{RHM}_{m i t}^{o} \quad$ Percentage of disrupted holding capacity of production center $m$ for $i$-type raw material in period $t$ in the incidence of disturbance $o$ (Percentage)

$\mathrm{RHFM}_{\text {mlit }}^{o} \quad$ Percentage of disrupted holding capacity of fortified production center $m$ at level $l$ for $i$-type raw material in period $t$ in the incidence of disturbance $o$ (Percentage)

$\mathrm{RDD}_{d p t}^{o} \quad$ Percentage of disrupted delivery capacity of distribution center $d$ for the $p$-type product in period $t$ in the incidence of disturbance $o$ (Percentage)

$\omega_{\text {swrt }}^{o} \quad$ A binary parameter, equal to 1 if route $r$ between primary supplier $s$ and warehouse $w$ is disrupted in period $t$ in the incidence of disturbance $o$; 0 , otherwise

$\eta_{w m r t}^{o} \quad$ A binary parameter, equal to 1 if route $r$ between warehouse $w$ and production center $m$ is disrupted in period $t$ in the incidence of disturbance $o$; 0, otherwise

$\psi_{m d r t}^{o}$

$\gamma_{d c r t}^{o}$ A binary parameter, equal to 1 if route $r$ between production center $m$ and distribution center $d$ is disrupted in period $t$ in the incidence of disturbance $o$; 0 , otherwise

A binary parameter, equal to 1 if route $r$ between distribution center $d$ and products first market $c$ is disrupted in period $t$ in the incidence of disturbance $o$; 0 , otherwise

$\mathrm{LW}_{w o}$

$\mathrm{LM}_{m o}$

$\mathrm{LD}_{d o}$

$\mathrm{DC}_{p c t}^{o}$

$\alpha_{s i}$

$\iota_{b i}$ $\mathrm{NCr}$ threshold for warehouse $w$ in the incidence of disturbance $o$ (Ton)

$\mathrm{NCr}$ threshold for production center $m$ in the incidence of disturbance $o$ (Ton)

$\mathrm{NCr}$ threshold for distribution center $d$ in the incidence of disturbance $o$ (Ton)

Demand of $p$-type product at first market $c$ in period $t$ in the incidence of disturbance $o$ (Ton)

Defective rate of primary supplier $s$ for providing $i$-type raw material (Percentage)

Defective rate of backup supplier $b$ for providing $i$-type raw material (Percentage)

Quantity of $i$-type raw material required for manufacturing unit $p$-type product by production technology $v$ (Ton)

$\lambda \quad$ Variability weight

$\xi \quad$ Risk aversion weight (RAW)

BM Sufficient large positive number

$\mathrm{UM}_{m t}^{o} \quad$ Percentage of operational capacity utilization at production center $m$ in period $t$ in the incidence of disturbance $o$ (Percentage)

$\pi_{o} \quad$ Probability of occurrence for disruption $o$ (Percentage)

\section{Environmental parameters}

$\mathrm{EOM}_{m} \quad$ Unit environmental impact associated with opening production center $m(\mathrm{Pt})$

$\mathrm{EOW}_{w}$

$\mathrm{EOD}_{d}$

$\mathrm{EPM}_{p v m t}^{o}$

Unit environmental impact associated with opening warehouse $w(\mathrm{Pt})$

Unit environmental impact associated with opening distribution center $d(\mathrm{Pt})$

Unit environmental impact of manufacturing $p$-type product by production technology $v$ at production center $m$ in period $t$ in the incidence of disturbance $o(\mathrm{Pt} / \mathrm{Ton})$

$\mathrm{EDD}_{\text {pdcrt }}^{o}$

Unit environmental impact of distributing of $p$-type product by distribution center $d$ to products first market $c$ by route $r$ in period $t$ in the incidence of disturbance $o$ (Pt/Ton.Km)

$\mathrm{ETSW}_{\text {iswrt }}^{o}$ Unit environmental impact of shipping $i$-type raw material from the primary supplier $s$ to warehouse $w$ by route $r$ in period $t$ in the incidence of disturbance $o$ (Pt/Ton.Km) 


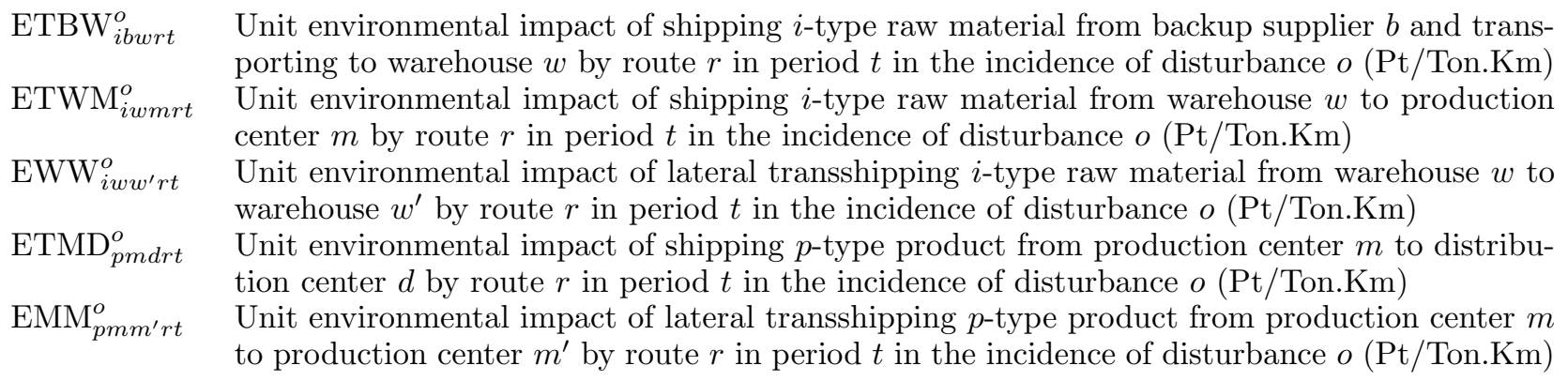

\section{Decision variables}

\footnotetext{
$\mathrm{OD}_{d}$

$\mathrm{OW}_{w}$

$\mathrm{OM}_{m}$

$\mathrm{VFS}_{s l}$

$\mathrm{VFM}_{m l}$

$\mathrm{CB}_{b}$

$\mathrm{SW}_{\text {swrt }}^{o}$

$\mathrm{WM}_{w m r t}^{o}$

$\mathrm{MD}_{m d r t}^{o}$

$\mathrm{DC}_{d c r t}^{o}$

$\mathrm{CD}_{d}^{\prime}$
$\mathrm{CM}_{m}^{\prime}$
$\mathrm{CW}_{w}^{\prime}$
$\mathrm{SSW}_{i p s w r t}^{o}$

SFSW $_{\text {ipswrt }}^{o}$

$\mathrm{SBW}_{\text {ipbwrt }}^{o}$

$\mathrm{SWM}_{\text {ipwmrt }}^{o}$

$\mathrm{IW}_{i p w t}^{o}$

$\mathrm{SWW}_{i p w w^{\prime} r t}^{o}$

$\mathrm{SMM}_{p m m^{\prime} r t}^{O}$

$\mathrm{SFMM}_{p m m^{\prime} r t}^{o}$

$\mathrm{PM}_{p m v t}^{o}$

$\mathrm{IMI}_{\text {ipmt }}^{o}$

$\mathrm{SMD}_{p m d r t}^{o}$

Indicates 1 if distribution center $d$ is established; 0, otherwise

Indicates 1 if warehouse $w$ is established; 0 , otherwise

Indicates 1 if production center $m$ is established; 0, otherwise

Indicates 1 if primary supplier $s$ is fortified at level $l ; 0$, otherwise

Indicates 1 if production center $m$ is fortified at level $l$; 0, otherwise

Indicates 1 if backup supplier $b$ is selected; 0, otherwise

Indicates 1 if warehouse $w$ is allocated to primary supplier $s$ by route $r$ in period $t$ in the incidence of disturbance $o$; 0, otherwise

Indicates 1 if production center $m$ is allocated to warehouse $w$ by route $r$ in period $t$ in the incidence of disturbance $o$; 0 , otherwise

Indicates 1 if distribution center $d$ is allocated to production center $m$ by route $r$ in period $t$ in the incidence of disturbance $o$; 0 , otherwise

Indicates 1 if products first market $c$ is allocated to distribution center $d$ by route $r$ in period $t$ in the incidence of disturbance $o$; 0 , otherwise

Indicates 1 if distribution center $d$ is a critical node; 0 , otherwise

Indicates 1 if production center $m$ is a critical node; 0 , otherwise

Indicates 1 if warehouse $w$ is a critical node; 0, otherwise

Quantity of $i$-type raw material of $p$-type product transported from primary supplier $s$ to warehouse $w$ by route $r$ in period $t$ in the incidence of disturbance $o$ (Ton)

Quantity of $i$-type raw material of $p$-type product transported from fortified primary supplier $s$ to warehouse $w$ by route $r$ in period $t$ in the incidence of disturbance $o$ (Ton)

Quantity of $i$-type raw material of $p$-type product transported from backup supplier $b$ to warehouse $w$ by route $r$ in period $t$ in the incidence of disturbance $o$ (Ton)

Quantity of $i$-type raw material of $p$-type product transported from warehouse $w$ to production center $m$ by route $r$ in period $t$ in the incidence of disturbance $o$ (Ton)

Inventory level of $i$-type raw material of $p$-type product at warehouse $w$ in period $t$ in the incidence of disturbance $o$ (Ton)

Quantity of $i$-type raw material of $p$-type product transported from warehouse $w$ to warehouse $w^{\prime}$ by route $r$ in period $t$ in the incidence of disturbance $o$ (Ton)

Quantity of $p$-type product transported from production center $m$ to production center $m^{\prime}$ by route $r$ in period $t$ in the incidence of disturbance $o$ (Ton)

Quantity of $p$-type product transported from fortified production center $m$ to production center $m^{\prime}$ by route $r$ in period $t$ in the incidence of disturbance $o$ (Ton)

Quantity of $p$-type product manufactured at production center $m$ by production technology $v$ in period $t$ in the incidence of disturbance $o$ (Ton)

Inventory level of the $i$-type raw material of $p$-type product at production center $m$ in period $t$ in the incidence of disturbance $o$ (Ton)

Quantity of $p$-type product transported from production center $m$ to distribution center $d$ by route $r$ in period $t$ in the incidence of disturbance $o$ (Ton)
} 
$\mathrm{SFMD}_{p m d r t}^{o}$ Quantity of $p$-type product transported from fortified production center $m$ to distribution center $d$ by route $r$ in period $t$ in the incidence of disturbance $o$ (Ton)

$\mathrm{SDC}_{p d c r t}^{o}$ Quantity of $p$-type product transported from distribution center $d$ to products first market $c$ by route $r$ in period $t$ in the incidence of disturbance $o$ (Ton)

$\mathrm{SHC}_{p c t}^{o}$

PSFS $_{i p s t}^{O}$

$\operatorname{PSFM}_{p m t}^{o}$

$\mathrm{UPSW}_{\text {ipswt }}^{o}$

Lost sale of $p$-type product at first market $c$ in period $t$ in the incidence of disturbance $o$ (Ton)

Pre-positioned EI level of $i$-type raw material of $p$-type product at the primary supplier $s$ in period $t$ in the incidence of disturbance $o$ (Ton)

Pre-positioned EI level of $p$-type product at the production center $m$ in period $t$ in the incidence of disturbance $o$ (Ton)

$\mathrm{UPMD}_{p m d t}^{o}$ Quantity of $i$-type raw material of $p$-type product purchased by warehouse $w$ from the EI of primary supplier $s$ in period $t$ in the incidence of disturbance $o$ (Ton)

Quantity of $p$-type product purchased by distribution center $d$ from the EI of production center $m$ in period $t$ in the incidence of disturbance $o$ (Ton)

\subsection{Network non-resiliency measures}

\subsubsection{Flow complexity}

FC investigates the overall interaction between network nodes, and the SC complexity considerably increases as FC rises [48]. Raising this measure makes SCM more perplexing and sophisticated, such that the system's resilience to avoid or mitigate disturbances will deteriorate, as will the required time and cost of recovery and the restoration quality. Consequently, critical risks can cause system failure and irreparable losses [36]. Equations (3.1) and (3.2) represent the total existing links between the investigated SC nodes.

$$
\sum_{(s, w, r, t, o)} \mathrm{SW}_{s w r t}^{o}+\sum_{(w, m, r, t, o)} \mathrm{WM}_{w m r t}^{o}+\sum_{(m, d, r, t, o)} \mathrm{MD}_{m d r t}^{o}+\sum_{(d, c, r, t, o)} \mathrm{DC}_{d c r t}^{o}
$$

$\mathrm{SW}_{s w r t}^{o}, \mathrm{WM}_{w m r t}^{o}, \mathrm{MD}_{m d r t}^{o}, \mathrm{DC}_{d c r t}^{o} \in\{0,1\}$

$\forall s \in S, \forall w \in W, \forall m \in M, \forall d \in D$ $\forall c \in C, \forall r \in R, \forall t \in T, \forall o \in O$.

\subsubsection{Node complexity}

$\mathrm{NC}$ indicates the total opened facilities or activated nodes in the network. Indeed, increasing the number of opened facilities elevates the NC measure. The described measure is assessed using equations (3.3) and (3.4) $[36,48]$.

$$
\begin{aligned}
& \sum_{w \in W} \mathrm{OW}_{w}+\sum_{m \in M} \mathrm{OM}_{m}+\sum_{d \in D} \mathrm{OD}_{d} \\
& \mathrm{OW}_{w}, \mathrm{OM}_{m}, \mathrm{OD}_{d} \in\{0,1\}
\end{aligned}
$$

$$
\forall w \in W, \forall m \in M, \forall d \in D
$$

\subsubsection{Node criticality}

The NCr measure is based on the overall flows to the network nodes. If the total output and input flow to a specific node of the SC exceed a specified threshold, the node under consideration is evaluated critically. TNRN is directly affected by increasing the number of critical network nodes that leads to a remarkable loss in the system's resilience capabilities during disturbances [36,48]. Equations $(3.5)-(3.8)$ represent the NCr measure for warehouses, production centers, and distribution centers, respectively.

$$
\mathrm{CW}_{w}^{\prime}=1 \mid \begin{aligned}
& \sum_{(i, p, s, r, t)} \mathrm{SSW}_{i p s w r t}^{o}+\sum_{(i, p, s, r, t)} \mathrm{SFSW}_{i p s w r t}^{o}+\sum_{(i, p, b, r, t)} \mathrm{SBW}_{i p b w r t}^{o} \\
& +\sum_{\left(i, p, w^{\prime} \in W /\{w\}, r, t\right)} \mathrm{SWW}_{i p w^{\prime} w r t}^{o}+\sum_{(i, p, s, t)} \mathrm{UPSW}_{i p s w t}^{o} \\
& +\sum_{(i, p, m, r, t)} \mathrm{SWM}_{i p w m r t}^{o}+\sum_{\left(i, p, w^{\prime} \in W /\{w\}, r, t\right)} \mathrm{SWW}_{i p w w^{\prime} r t}^{o} \geq \mathrm{LW}_{w o}
\end{aligned} \quad \forall w \in W, \forall o \in O
$$




$$
\begin{aligned}
& \mathrm{CM}_{m}^{\prime}=1 \mid \begin{array}{l}
\sum_{(i, p, w, r, t)} \mathrm{SWM}_{i p w m r t}^{o}+\sum_{\left(p, m^{\prime} \in M /\{m\}, r, t\right)} \mathrm{SMM}_{p m^{\prime} m r t}^{o} \\
+\sum_{\left(p, m^{\prime} \in M /\{m\}, r, t\right)} \operatorname{SFMM}_{p m^{\prime} m r t}^{o}+\sum_{(p, v, t)} \mathrm{PM}_{p m v t}^{o} \delta_{i p v} \\
+\sum_{\left(p, m^{\prime} \in M /\{m\}, r, t\right)} \operatorname{SMM}_{p m m^{\prime} r t}^{o}+\sum_{\left(p, m^{\prime} \in M /\{m\}, r, t\right)} \operatorname{SFMM}_{p m m^{\prime} r t}^{o} \\
\sum_{(p, d, r, t)} \mathrm{SMD}_{p m d r t}^{o}+\sum_{(p, d, r, t)} \operatorname{SFMD}_{p m d r t}^{o}+\sum_{(p, d, t)}^{o} \mathrm{UPMD}_{p m d t}^{o} \geq \mathrm{LM}_{m o}
\end{array} \quad \forall m \in M, \forall o \in O \\
& \mathrm{CD}_{d}^{\prime}=1 \mid \begin{array}{l}
\sum_{(p, m, r, t)} \mathrm{SMD}_{p m d r t}^{o}+\sum_{(p, m, r, t)} \mathrm{SFMD}_{p m d r t}^{o}+\sum_{(p, m, t)} \mathrm{UPMD}_{p m d t}^{o} \\
+\sum_{(p, c, r, t)} \mathrm{SDC}_{p d c r t}^{o} \geq \mathrm{LD}_{d o}
\end{array} \quad \forall d \in D, \forall o \in O
\end{aligned}
$$

$\mathrm{CW}_{w}^{\prime}, \mathrm{CM}_{m}^{\prime}, \mathrm{CD}_{d}^{\prime} \in\{0,1\}$

$\forall w \in W, \forall m \in M$, $\forall d \in D$.

\subsection{Objective functions}

\subsubsection{Total cost}

The OF (3.9) ensures the minimization of the expected costs of SC under disruption scenarios. This term includes the costs of establishing new facilities and contracting with backup facilities (TFOC), product transshipment $\left(\mathrm{TTC}_{o}\right)$, product distribution $\left(\mathrm{TDC}_{o}\right)$, manufacturing operations $\left(\mathrm{TOC}_{o}\right)$, lost sales $\left(\mathrm{TSHC}_{o}\right)$, and holding inventory $\left(\mathrm{THC}_{o}\right)$ as permanent costs. Besides, this $\mathrm{OF}$ entails the structural resiliency cost, consisting of the costs of facilities' fortification (TFFC), maintaining pre-positioned $\mathrm{EI}\left(\mathrm{TPIC}_{o}\right)$, purchasing from the EI kept by the fortified facilities $\left(\mathrm{TPEIC}_{o}\right)$, and penalty for the remaining unused capacity $\left(\mathrm{TPUC}_{o}\right)$. Terms (3.10)-(3.19) define the components of the total SC costs.

$$
\begin{aligned}
\mathrm{Min} \mathrm{TC}= & \sum_{o \in O} \pi_{o}\left(\begin{array}{l}
\mathrm{TFOC}+\mathrm{TTC}_{o}+\mathrm{TDC}_{o}+\mathrm{TOC}_{o}+\mathrm{THC}_{o} \\
+\mathrm{TSHC}_{o}+\mathrm{TFFC}+\mathrm{TPIC}_{o}+\mathrm{TPEIC}_{o}+\mathrm{TECC}_{o}
\end{array}\right) \\
\mathrm{TFOC}= & \left(\sum_{w \in W} \mathrm{FOW}_{w} \mathrm{OW}_{w}+\sum_{m \in M} \mathrm{FOM}_{m} \mathrm{OM}_{m}+\sum_{d \in D} \mathrm{FOD}_{d} \mathrm{OD}_{d}+\sum_{b \in B} \mathrm{FCB}_{b} \mathrm{CB}_{b}\right) \\
\mathrm{TTC}_{o}= & \sum_{(i, p, s, w, r, t)} \frac{\mathrm{TSW}_{i s w r t}^{o}\left(\mathrm{SSW}_{i p s w r t}^{o}+\mathrm{SFSW}_{i p s w r t}^{o}\right) d_{s w r}}{\left(1-\alpha_{s i}\right)}+\sum_{(i, p, b, w, r, t)} \frac{\mathrm{TBW}_{i b w r t} \mathrm{SBW}_{i p b w r t}^{o} d_{b w r}}{\left(1-\iota_{b i}\right)} \\
& \times \sum_{(i, p, w, m, r, t)} \operatorname{TWM}_{i w m r t}^{o} \mathrm{SWM}_{i p w m r t}^{o} d_{w m r}+\mathrm{TWW}_{i p w w^{\prime} r t} \mathrm{SWW}_{i p w w^{\prime} r t}^{o} d_{w w^{\prime} r} \\
& +\sum_{\left(p, m, d, w, w^{\prime} \in W /\{w\}, r, t\right)} \mathrm{TMD}_{p m d r t}^{o}\left(\mathrm{SMD}_{p m d r t}^{o}+\mathrm{SFMD}_{p m d r t}^{o}\right) d_{m d r} \\
& +\sum_{\left(p, m, m^{\prime} \in M /\{m\}, r, t\right)} \mathrm{TMM}_{p m m^{\prime} r t}^{o}\left(\mathrm{SMM}_{p m m^{\prime} r t}^{o}+\mathrm{SFMM}_{p m m^{\prime} r t}^{o}\right) d_{m m^{\prime} r} \\
\mathrm{TDC}_{o}= & \sum_{(p, d, c, r, t)} \mathrm{DCD}_{p d c r t}^{o} \mathrm{SDC}_{p d c r t}^{o} d_{d c r} \\
\mathrm{TOC}_{o}= & \sum_{p \in P} \sum_{m \in M} \sum_{v \in V} \sum_{t \in T} \mathrm{PC}_{p m v t}^{o} \mathrm{PM}_{p m v t}^{o} \\
\mathrm{TSHC}_{o}= & \sum_{p \in P} \sum_{c \in C} \sum_{t \in T} \mathrm{SCC}_{p c t}^{o} \mathrm{SHC}_{p c t}^{o} \\
\mathrm{THC}_{o}= & \sum_{(i, p, w, t)} \mathrm{HCW}_{i w t}^{o} \mathrm{IW}_{i p w t}^{o}+\sum_{(i, p, m, t)} \mathrm{HCMI}_{i p m t}^{o} \mathrm{IMI}_{i p m t}^{o}
\end{aligned}
$$




$$
\begin{aligned}
& \mathrm{TFFC}=\sum_{(s, l)} \mathrm{FFS}_{s l} \mathrm{VFS}_{s l}+\sum_{(m, l)} \mathrm{FFM}_{m l} \mathrm{VFM}_{m l} \\
& \mathrm{TPIC}_{o}=\sum_{(i, p, s, t)} \operatorname{PIFS}_{i s t}^{o} \mathrm{PSFS}_{i p s t}^{o}+\sum_{(p, m, t)} \mathrm{PIFM}_{p m t}^{o} \mathrm{PSFM}_{p m t}^{o} \\
& \mathrm{TPEIC}_{o}=\sum_{(i, p, s, w, t)} \mathrm{BESW}_{i s w t}^{o} \mathrm{UPSW}_{i p s w t}^{o}+\sum_{(p, m, d, t)} \mathrm{BEMD}_{p m d t}^{o} \mathrm{UPMD}_{p m d t}^{o} \\
& \mathrm{TPUC}_{o}=\sum_{o \in O} \beta_{o}\left(\begin{array}{l}
\sum_{(i, w, t)}\left(\begin{array}{l}
\left(1-\mathrm{RSW}_{w i t}^{o}\right) S c a p w_{w i} \mathrm{OW}_{w}-\sum_{(p, s, r)} \mathrm{SSW}_{i p s w r t}^{o}-\sum_{(p, s, r)} \mathrm{SFSW}_{i p s w r t}^{o} \\
-\sum_{\left(p, \forall w^{\prime} \in W /\{w\}, r\right)} \mathrm{SWW}_{i p w^{\prime} w r t}^{o}-\sum_{(p, b, r)} \mathrm{SBW}_{i p b w r t}^{o}
\end{array}\right) \\
+\sum_{(p, m, v, t)}\left(\begin{array}{l}
\left(\left(\mathrm{OM}_{m}-\sum_{l \in L} \mathrm{VFM}_{m l}\right)\left(1-\mathrm{RPM}_{m p v t}^{o}\right)\right. \\
\left.+\sum_{l \in L} \mathrm{VFM}_{m l}\left(1-\mathrm{RPFM}_{m l p v t}^{o}\right)\right) \\
P c a p m_{m p v} \mathrm{UM}_{m t}^{o}-\mathrm{PM}_{p m t}^{o}
\end{array}\right. \\
\sum_{(p, d, t)}\left(\begin{array}{l}
\left(1-\mathrm{RDD}_{d p t}^{o}\right) \operatorname{Dcapd}_{d p} \mathrm{OD}_{d}-\sum_{(m, r)} \mathrm{SMD}_{p m d r t}^{o}-\sum_{(m, r)} \mathrm{SFMD}_{p m d r t}^{o}
\end{array}\right)
\end{array}\right) .
\end{aligned}
$$

\subsubsection{Total environmental impact}

The OF (3.20) minimizes the expected TEI entailing emissions and hazards associated with establishing new facilities (TEE), product transmission $\left(\mathrm{TTE}_{o}\right)$, and distribution and manufacturing of products $\left(\mathrm{TOE}_{o}\right)$ defined by terms (3.21)-(3.23).

$$
\begin{aligned}
\mathrm{Min} \mathrm{TEI}= & \sum_{o \in O} \pi_{o}\left(\mathrm{TEE}+\mathrm{TTE}_{o}+\mathrm{TOE}_{o}\right) \\
\mathrm{TEE}= & \sum_{w \in W} \mathrm{EOW}_{w} \mathrm{OW}_{w}+\sum_{m \in M} \mathrm{EOM}_{m} \mathrm{OM}_{m}+\sum_{d \in D} \mathrm{EOD}_{d} \mathrm{OD}_{d} \\
\mathrm{TOE}_{o}= & \sum_{(p, v, m, t)} \mathrm{EPM}_{p v m t}^{o} \mathrm{PM}_{p m v t}^{o}+\sum_{(p, d, c, r, t)} \mathrm{EDD}_{p d c r t}^{o} \mathrm{SDC}_{p d c r t}^{o} d_{d c r} \\
\mathrm{TTE}_{o}= & \sum_{(i, p, s, w, r, t)} \frac{\mathrm{ESW}_{i s w r t}^{o}\left(\mathrm{SSW}_{i p s w r t}^{o}+\mathrm{SFSW}_{i p s w r t}^{o}\right) d_{s w r}}{1-\alpha_{s i}}+\sum_{(i, p, w, m, r, t)} \mathrm{EWM}_{i w m r t}^{o} \mathrm{SWM}_{i p w m r t}^{o} d_{w m r} \\
& +\sum_{(p, m, d, r, t)} \sum_{\mathrm{EMD}_{p m d r t}^{o}\left(\mathrm{SMD}_{p m d r t}^{o}+\mathrm{SFMD}_{p m d r t}^{o}\right) d_{m d r}+\sum_{\left(i, p, w, w^{\prime} \in W /\{w\}, r, t\right)} \mathrm{EWW}_{i p w w^{\prime} r t}^{o}} \\
& \times \mathrm{SWW}_{i p w w^{\prime} r t}^{o} d_{w w^{\prime} r}+\sum_{\left(p, m, m^{\prime} \in M /\{m\}, r, t\right)} \mathrm{EMM}_{p m m^{\prime} r t}^{o}\left(\mathrm{SMM}_{p m m^{\prime} r t}^{o}+\mathrm{SFMM}_{p m m^{\prime} r t}^{o}\right) d_{m m^{\prime} r} \\
& +\sum_{(i, p, b, w, r, t)} \frac{\mathrm{EBW}_{i b w r t}^{o} \mathrm{SBW}_{i p b w r t}^{o} d_{b w r}}{1-\iota_{b i}}
\end{aligned}
$$

\subsubsection{Total non-resiliency of the network}

The OF (3.20) minimizes the TNRN of the investigated SC, including FC, NC, and NCr. The network non-resiliency measures are comprehensively described in Section 3.3.

$$
\operatorname{TNRES}=\left(\begin{array}{l}
\sum_{o} \pi_{o}\left(\sum_{(s, w, r, t, o)} \phi \mathrm{SW}_{s w r t}^{o}+\sum_{(w, m, r, t, o)} \phi^{\prime} \mathrm{WM}_{w m r t}^{o}\right. \\
\left.+\sum_{(m, d, r, t, o)} \phi^{\prime \prime} \mathrm{MD}_{m d r t}^{o}+\sum_{(d, c, r, t, o)} \phi^{\prime \prime \prime} \mathrm{DC}_{d c r t}^{o}\right) \\
+\left(\sum_{w} \mu^{\prime} \mathrm{OW}_{w}+\sum_{m} \mu^{\prime \prime} \mathrm{OM}_{m}+\sum_{d} \mu^{\prime \prime \prime} \mathrm{OD}_{d}\right) \\
+\left(\sum_{w} \theta^{\prime} \mathrm{CW}_{w}^{\prime}+\sum_{m} \theta^{\prime \prime} \mathrm{CM}_{m}^{\prime}+\sum_{d} \theta^{\prime \prime \prime} \mathrm{CD}_{d}^{\prime}\right)
\end{array}\right)
$$




\subsection{Constraints}

\section{Disrupted routes' selection considerations}

Constraints (3.25)-(3.28) stipulate the condition of choosing a maximum of one route between two facilities in a particular period.

$$
\begin{array}{ll}
\sum_{r \in R} \mathrm{SW}_{\text {swrt }}^{o} \leq 1 & \forall s \in S, \forall w \in W, \forall t \in T, \forall o \in O \\
\sum_{r \in R} \mathrm{WM}_{w m r t}^{o} \leq 1 & \forall w \in W, \forall m \in M, \forall t \in T, \forall o \in O \\
\sum_{r \in R} \mathrm{MD}_{m d r t}^{o} \leq 1 & \forall m \in M, \forall d \in D, \forall t \in T, \forall o \in O \\
\sum_{r \in R} \mathrm{DC}_{d c r t}^{o} \leq 1 & \forall d \in D, \forall c \in C, \forall t \in T, \forall o \in O .
\end{array}
$$

Conditions for allocation of facilities in case of capacity and route disruption

Constraints (3.29)-(3.34) enforce that the allocation of two facilities through a specific route is possible only if they are opened and there is no disruption associated with that route. Constraints (3.35)-(3.38) stipulate that establishing a flow between two facilities through a specific route depends on allocating these two facilities to each other through that route.

$$
\begin{array}{ll}
\mathrm{SW}_{s w r t}^{o} \leq \mathrm{OW}_{w}\left(1-\omega_{s w r t}^{o}\right) & \forall s \in S, \forall w \in W, \forall r \in R, \forall t \in T, \forall o \in O \\
\mathrm{WM}_{w m r t}^{o} \leq \mathrm{OW}_{w}\left(1-\eta_{w m r t}^{o}\right) & \forall w \in W, \forall m \in M, \forall r \in R, \forall t \in T, \forall o \in O \\
\mathrm{WM}_{w m r t}^{o} \leq \mathrm{OM}_{m}\left(1-\eta_{w m r t}^{o}\right) & \forall w \in W, \forall m \in M, \forall r \in R, \forall t \in T, \forall o \in O \\
\mathrm{MD}_{m d r t}^{o} \leq \mathrm{OM}_{m}\left(1-\psi_{m d r t}^{o}\right) & \forall m \in M, \forall d \in D, \forall r \in R, \forall t \in T, \forall o \in O \\
\mathrm{MD}_{m d r t}^{o} \leq \mathrm{OD}_{d}\left(1-\psi_{m d r t}^{o}\right) & \forall m \in M, \forall d \in D, \forall r \in R, \forall t \in T, \forall o \in O \\
\mathrm{DC}_{d c r t}^{o} \leq \mathrm{OD}_{d}\left(1-\gamma_{d c r t}^{o}\right) & \forall d \in D, \forall c \in C, \forall r \in R, \forall t \in T, \forall o \in O \\
\sum_{(i, p)} \mathrm{SSW}_{i p s w r t}^{o}+\sum_{(i, p)} \mathrm{SFSW}_{i p s w r t}^{o} \leq \mathrm{SW}_{s w r t}^{o} \mathrm{BM} & \forall s \in S, \forall w \in W, \forall r \in R, \forall t \in T, \forall o \in O \\
\sum_{(i, p)} \mathrm{SWM}_{i p w m r t}^{o} \leq \mathrm{WM}_{w m r t}^{o} \mathrm{BM} & \forall w \in W, \forall m \in M, \forall r \in R, \forall t \in T, \forall o \in O \\
\sum_{p \in P} \mathrm{SMD}_{p m d r t}^{o}+\sum_{p \in P} \mathrm{SFMD}_{p m d r t}^{o} \leq \mathrm{MD}_{m d r t}^{o} \mathrm{BM} & \forall m \in M, \forall d \in D, \forall r \in R, \forall t \in T, \forall o \in O \\
\sum_{p \in P} \mathrm{SDC}_{p d c r t}^{o} \leq \mathrm{DC}_{d c r t}^{o} \mathrm{BM} &
\end{array}
$$

\section{Disrupted capacity constraints}

Constraints (3.39)-(3.48) illustrate capacity constraints for primary suppliers, backup suppliers, warehouses, production centers, and distribution centers during disturbances, respectively. Note that the condition of opening facilities, the percentage of disrupted capacity, the defective rate of facilities in the incidence of disruption, and decisions about facilities' fortification at a particular level are considered in the mentioned terms. Constraints (3.43), (3.46), and (3.47) are holding capacity equations, and the other equations stipulate operational limitations.

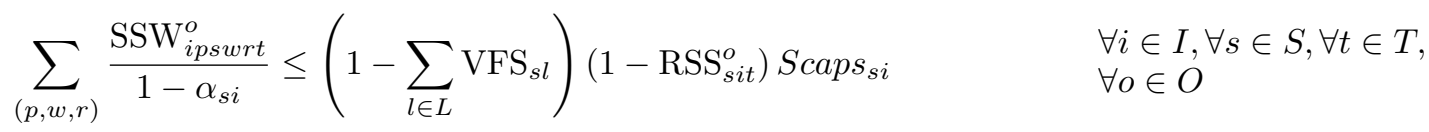




$$
\begin{aligned}
& \sum_{(p, w, r)} \frac{\mathrm{SFSW}_{i p s w r t}^{o}}{1-\alpha_{s i}} \leq \sum_{l \in L} \operatorname{VFS}_{s l}\left(1-\mathrm{RSFS}_{\text {slit }}^{o}\right) \text { Scaps }_{s i} \\
& \sum_{(p, w, r)} \frac{\mathrm{SBW}_{i p b w r t}^{o}}{1-\iota_{b i}} \leq S c a p b_{b i}^{o} \mathrm{CB}_{b} \\
& \sum_{(p, s, r)} \mathrm{SSW}_{\text {ipswrt }}^{o}+\sum_{(p, s, r)} \mathrm{SFSW}_{\text {ipswrt }}^{o}+\sum_{\left(p, \forall w^{\prime} \in W /\{w\}, r\right)} \mathrm{SWW}_{\text {ipw }}^{o} w r t \\
& +\sum_{(p, b, r)} \mathrm{SBW}_{i p b w r t}^{o} \leq\left(1-\mathrm{RSW}_{w i t}^{o}\right) S_{c a p w_{w i}} \mathrm{OW}_{w} \\
& \sum_{p \in P} \mathrm{IW}_{i p w t}^{o} \leq\left(1-\mathrm{RHW}_{i w t}^{o}\right) H c a p w_{w i} \mathrm{OW}_{w} \\
& \mathrm{PM}_{p m v t}^{o} \leq P c a p m_{m v p} \mathrm{UM}_{m t}^{o} \sum_{l \in L} \mathrm{VFM}_{m l}\left(1-\mathrm{RPFM}_{m l p v t}^{o}\right) \\
& \mathrm{PM}_{p m v t}^{o} \leq \operatorname{Pcapm}_{m v p} \mathrm{UM}_{m t}^{o}\left(\mathrm{OM}_{m}-\sum_{l \in L} \mathrm{VFM}_{m l}\right)\left(1-\mathrm{RPM}_{m p v t}^{o}\right) \\
& \sum_{p \in P} \mathrm{IMI}_{i p m t}^{o} \leq \text { Hcapmi }_{m i} \sum_{l \in L} \operatorname{VFM}_{m l}\left(1-\mathrm{RHFM}_{m l i t}^{o}\right) \\
& \sum_{p \in P} \mathrm{IMI}_{i p m t}^{o} \leq \text { Hcapmi }_{m i}\left(\mathrm{OM}_{m}-\sum_{l \in L} \mathrm{VFM}_{m l}\right)\left(1-\mathrm{RHM}_{m i t}^{o}\right) \\
& \sum_{(m, r)} \mathrm{SMD}_{p m d r t}^{o}+\sum_{(m, r)} \mathrm{SFMD}_{p m d r t}^{o} \leq\left(1-\mathrm{RDD}_{d p t}^{o}\right) \operatorname{Dcapd}_{d p} \mathrm{OD}_{d}
\end{aligned}
$$

\section{Flow balance equations}

Constraints (3.49) and (3.50) enforce a flow balance for production centers and distribution centers, respectively.

$$
\begin{array}{ll}
\sum_{(d, r)} \mathrm{SMD}_{p m d r t}^{o}+\sum_{(d, r)} \mathrm{SFMD}_{p m d r t}^{o}=\sum_{v \in V} \mathrm{PM}_{p m v t}^{o} & \forall p \in P, \forall m \in M, \forall t \in T \\
\sum_{(m, r)} \mathrm{SMD}_{p m d r t}^{o}+\sum_{(m, r)} \mathrm{SFMD}_{p m d r t}^{o}+\sum_{m \in M} \mathrm{UPMD}_{p m d t}^{o}=\sum_{(c, r)} \mathrm{SDC}_{p d c r t}^{o} & \begin{array}{l}
\forall p \in P, \forall d \in D, \forall t \in T \\
\forall o \in O .
\end{array}
\end{array}
$$

\section{Inventory balance equations}

Constraints (3.51) and (3.52) represent inventory balance equations for warehouses and production centers, respectively.

$$
\begin{array}{cl}
\mathrm{IW}_{i p w t}^{o}=\mathrm{IW}_{i p w t-1}^{o}+\sum_{(s, r)} \mathrm{SSW}_{\text {ipswrt }}^{o}+\sum_{(s, r)} \mathrm{SFSW}_{i p s w r t}^{o} & \\
+\sum_{\left(w^{\prime} \in W /\{w\}, t\right)} \mathrm{SWW}_{i p w^{\prime} w r t}^{o}+\sum_{s \in S} \mathrm{UPSW}_{i p s w t}^{o}+\sum_{(b, r)} \mathrm{SBW}_{\text {ipbwrt }}^{o} & \forall i \in I, \forall p \in P, \forall w \in W, \\
\quad-\sum_{(m, r)} \mathrm{SWM}_{i p w m r t}^{o}-\sum_{\left(w^{\prime} \in W /\{w\}, t\right)} \mathrm{SWW}_{i p w w^{\prime} r t}^{o} & \forall t \in T, \forall o \in O \\
\mathrm{IMI}_{i p m t}^{o}=\mathrm{IMI}_{i p m t-1}^{o}+\sum_{(w, r)} \mathrm{SWM}_{i p w m r t}^{o}-\sum_{v \in V} \mathrm{PM}_{p m v t}^{o} \delta_{i p v} & \forall i \in I, \forall p \in P, \forall m \in M, \\
& \forall t \in T, \forall o \in O .
\end{array}
$$




\section{Demand fulfilment equations}

Constraint (3.53) ensures demand satisfaction for first markets.

$$
\begin{array}{ll}
\sum_{(d, r)} \mathrm{SDC}_{p d c r t}^{o}+\mathrm{SHC}_{p c t}^{o}=\mathrm{DC}_{p c t}^{o} & \forall p \in P, \forall c \in C, \forall t \in T, \\
& \forall o \in O .
\end{array}
$$

\section{Resiliency considerations}

Constraints (3.54), (3.55) ensure that the quantity of pre-positioned EI kept by fortified facilities cannot transgress their maximum holding capacities of pre-positioned EI. Constraints (3.56), (3.57) stipulate that the products purchased from the pre-positioned EI of fortified facilities must not exceed the quantity of prepositioned EI kept by them. Constraint (3.58) assures that a facility can only be fortified to a specified level once it has been established. Constraints (3.59), (3.60) ensure that primary suppliers and manufacturing centers can be fortified only at a single level. Non-linear terms (3.5)-(3.8) that respectively represent the non-criticality of nodes' primary suppliers, warehouses, production centers, and distribution centers, are substituted with corresponding linear constraints (3.61)-(3.66).

$$
\begin{aligned}
& \sum_{p \in P} \operatorname{PSFS}_{\text {ipst }}^{o} \leq \sum_{l \in L} \operatorname{MHFS}_{\text {slit }}^{o} \operatorname{VFS}_{s l} \\
& \mathrm{PSFM}_{p m t}^{o} \leq \sum_{l \in L} \mathrm{MHFM}_{m l p t}^{o} \mathrm{VFM}_{m l} \\
& \sum_{w \in W} \mathrm{UPSW}_{i p s w t}^{o} \leq \mathrm{PSFS}_{i p s t}^{o} \\
& \sum_{d \in D} \mathrm{UPMD}_{p m d t}^{o} \leq \mathrm{PSFM}_{p m t}^{o} \\
& \mathrm{VFM}_{m l} \leq \mathrm{OM}_{m} \\
& \sum_{l \in L} \operatorname{VFS}_{s l} \leq 1 \\
& \sum_{l \in L} \operatorname{VFM}_{m l} \leq 1 \\
& \sum_{(i, p, s, r, t)} \mathrm{SSW}_{i p s w r t}^{o}+\sum_{(i, p, w, r, t)} \mathrm{SFSW}_{i p s w r t}^{o}+\sum_{\left(i, p, w^{\prime} \in W /\{w\}, r, t\right)} \mathrm{SWW}_{i p w^{\prime} w r t}^{o} \\
& +\sum_{(i, p, s, t)}^{(i, p, s, r, t)} \mathrm{UPSW}_{i p s w t}^{o}+\sum_{\left(i, p, w^{\prime} \in W /\{w\}, r, t\right)}^{(i, p, w, r, t)} \mathrm{SWW}_{i p w w^{\prime} r t}^{o}+\sum_{(i, p, m, r, t)}^{\left(i, p, w^{\prime} \in W /\{w\}, r, t\right)} \mathrm{SWM}_{i p w m r t}^{o} \quad \forall w \in W, \forall o \in O \\
& \leq \mathrm{BMCW}_{w}^{\prime}+\mathrm{LW}_{w o} \\
& \sum_{(i, p, s, r, t)} \mathrm{SSW}_{i p s w r t}^{o}+\sum_{(i, p, w, r, t)} \mathrm{SFSW}_{i p s w r t}^{o}+\sum_{(i, p, s, t)} \mathrm{UPSW}_{i p s w t}^{o} \\
& +\sum_{\left(i, p, w^{\prime} \in W /\{w\}, r, t\right)} \mathrm{SWW}_{i p w^{\prime} w r t}^{o}+\sum_{\left(i, p, w^{\prime} \in W /\{w\}, r, t\right)} \mathrm{SWW}_{i p w w^{\prime} r t}^{o} \quad \forall w \in W, \forall o \in O \\
& +\sum_{(i, p, m, r, t)} \mathrm{SWM}_{i p w m r t}^{o}>\mathrm{LW}_{w o} \mathrm{CW}_{w}^{\prime} \\
& \forall i \in I, \forall s \in S, \forall t \in T, \\
& \forall o \in O \\
& \forall p \in P, \forall m \in M, \forall t \in T \text {, } \\
& \forall o \in O \\
& i \in I, \forall p \in P, \forall s \in S, \\
& \forall t \in T, \forall o \in O \\
& \forall p \in P, \forall m \in M, \forall t \in T \text {, } \\
& \forall o \in O \\
& \forall m \in M \\
& \forall s \in S \\
& \forall m \in M
\end{aligned}
$$




$$
\begin{aligned}
& \sum_{(i, p, w, r, t)} \mathrm{SWM}_{i p w m r t}^{o}+\sum_{\left(p, m^{\prime} \in M /\{m\}, r, t\right)} \mathrm{SMM}_{p m^{\prime} m r t}^{o} \\
& +\sum_{\left(p, m^{\prime} \in M /\{m\}, r, t\right)} \operatorname{SFMM}_{p m^{\prime} m r t}^{o}+\sum_{(p, v, t)}^{(i, p, w, r, t)} \mathrm{PM}_{p m v t}^{o} \delta_{i p v} \\
& +\sum_{\left(p, m^{\prime} \in M /\{m\}, r, t\right)} \mathrm{SMM}_{p m m^{\prime} r t}^{o}+\sum_{\left(p, m^{\prime} \in M /\{m\}, r, t\right)} \mathrm{SFMM}_{p m m^{\prime} r t}^{o} \quad \forall m \in M, \forall o \in O \\
& \sum_{(p, d, r, t)}^{\left.p, m^{\prime} \in M /\{m\}, r, t\right)} \mathrm{SMD}_{p m d r t}^{o}+\sum_{(p, d, r, t)} \mathrm{SFMD}_{p m d r t}^{o}+\sum_{(p, d, t)}^{\left(\mathrm{C}^{\prime}\right)} \mathrm{UPMD}_{p m d t}^{o} \\
& \leq \mathrm{BMCM}_{m}^{\prime}+\mathrm{LM}_{m o} \\
& \sum_{(i, p, w, r, t)} \mathrm{SWM}_{i p w m r t}^{o}+\sum_{\left(p, m^{\prime} \in M /\{m\}, r, t\right)} \mathrm{SMM}_{p m^{\prime} m r t}^{o}+\sum_{(p, v, t)} \mathrm{PM}_{p m v t}^{o} \delta_{i p v} \\
& +\sum_{\left(p, m^{\prime} \in M /\{m\}, r, t\right)} \mathrm{SFMM}_{p m^{\prime} m r t}^{o}+\sum_{\left(p, m^{\prime} \in M /\{m\}, r, t\right)} \mathrm{SMM}_{p m m^{\prime} r t}^{o} \\
& +\sum_{\left(p, m^{\prime} \in M /\{m\}, r, t\right)} \mathrm{SFMM}_{p m m^{\prime} r t}^{o}+\sum_{(p, d, r, t)}^{\left(p, m^{\prime} \in M /\{m\}, r, t\right)} \mathrm{SMD}_{p m d r t}^{o}+\sum_{(p, d, r, t)} \mathrm{SFMD}_{p m d r t}^{o} \\
& +\sum_{(p, d, t)} \mathrm{UPMD}_{p m d t}^{o}>\mathrm{LM}_{m o} \mathrm{CM}_{m}^{\prime} \\
& \sum_{(p, m, r, t)} \mathrm{SMD}_{p m d r t}^{o}+\sum_{(p, m, r, t)} \mathrm{SFMD}_{p m d r t}^{o}+\sum_{(p, m, t)} \mathrm{UPMD}_{p m d t}^{o} \\
& +\sum_{(p, c, r, t)} \mathrm{SDC}_{p d c r t}^{o} \leq \mathrm{BMCD}_{d}^{\prime}+\mathrm{LD}_{d o} \\
& \sum_{(p, m, r, t)} \mathrm{SMD}_{p m d r t}^{o}+\sum_{(p, m, r, t)} \mathrm{SFMD}_{p m d r t}^{o}+\sum_{(p, m, t)} \mathrm{UPMD}_{p m d t}^{o} \\
& +\sum_{(p, c, r, t)} \mathrm{SDC}_{p d c r t}^{o}>\mathrm{LD}_{d o} \mathrm{CD}_{d}^{\prime}
\end{aligned}
$$

\section{Remaining constraints}

Constraint (3.67) indicates the auxiliary term utilized in equation (4.13). Moreover, constraints (3.68)-(3.71) stipulate positive and binary decision variables.

$$
\begin{aligned}
& \left(\begin{array}{l}
\mathrm{TFOC}+\mathrm{TTC}_{o}+\mathrm{TDC}_{o}+\mathrm{TOC}_{o}+\mathrm{THC}_{o}+\mathrm{TSHC}_{o} \\
+\mathrm{TPIC}_{o}+\mathrm{TFFC}+\mathrm{TPEIC}_{o}+\mathrm{TECC}_{o}+\mathrm{TPUC}_{o}
\end{array}\right) \\
& -\sum_{o \in O} \pi_{o}\left(\begin{array}{l}
\mathrm{TFOC}^{+} \mathrm{TTC}_{o}+\mathrm{TDC}_{o}+\mathrm{TOC}_{o}+\mathrm{THC}_{o}+\mathrm{TSHC}_{o} \\
+\mathrm{TPIC}_{o}+\mathrm{TFFC}+\mathrm{TPEIC}_{o}+\mathrm{TECC}_{o}+\mathrm{TPUC}_{o}
\end{array}\right)+\tau_{o} \geq 0 \quad \forall o \in O \\
& \tau_{o} \geq 0 \\
& \mathrm{SSW}_{i p s w r t}^{o}, \mathrm{SFSW}_{\text {ipswrt }}^{o}, \mathrm{SWM}_{i p w m r t}^{o}, \mathrm{SWW}_{i p w w^{\prime} r t}^{o}, \mathrm{SMD}_{\text {pmdrt }}^{o} \text {, } \\
& \mathrm{SMM}_{p m m^{\prime} r t}^{o}, \mathrm{SFMD}_{p m d r t}^{o}, \mathrm{SFMM}_{p m m^{\prime} r t}^{o}, \mathrm{SDC}_{p d c r t}^{o}, \mathrm{PM}_{p m v t}^{o} \text {, } \\
& \mathrm{IMI}_{i p m t}^{o}, \mathrm{IW}_{i p w t}^{o}, \mathrm{SHC}_{p c t}^{o}, \mathrm{SHQ}_{i p q t}^{o} \geq 0 \\
& \forall o \in O \\
& \forall i \in I, \forall p \in P, \forall s \in S \text {, } \\
& \forall w \in W, \forall m \in M, \forall d \in D, \\
& \forall c \in C, \forall o \in O, \forall r \in R \text {, } \\
& \forall l \in L, \forall r \in R, \forall v \in V, \forall t \in T
\end{aligned}
$$




$$
\begin{aligned}
& \mathrm{OW}_{w}, \mathrm{OM}_{m}, \mathrm{OD}_{d}, \mathrm{SW}_{s w r t}^{o}, \mathrm{WM}_{w m r t}^{o}, \mathrm{MD}_{m d r t}^{o}, \\
& \mathrm{DC}_{d c r t}^{o}, \mathrm{CB}_{b}, C K_{k}^{\prime}, \mathrm{CW}_{w}^{\prime}, \mathrm{CM}_{m}^{\prime}, \mathrm{CD}_{d}^{\prime} \in\{0,1\}
\end{aligned}
$$

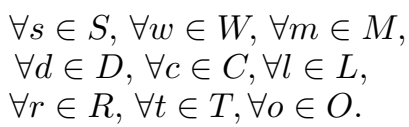

\section{SOLUTION APPROACH}

The model investigated in Section 3 is entangled with two issues: multiple OFs, and the destructive impact of operational risks associated with usual uncertainty and instability in the business environment. These problems in the proposed model are tackled by taking three measures:

- Multiple OFs are countered by applying an augmented $\varepsilon$-constraint method to the developed multi-objective TSSP model;

- An SBR formulation base on the Mulvey RO technique is applied to cope with operational risks.

\subsection{Applying multi-objective programming techniques}

Multi-objective programming is an efficient technique with demonstrated accomplishments to resolve multiobjective problems or counter the issue of multiple OFs by taking into account technical constraints. A problem can be formulated as multi-objective only in the case of a conflict between OFs. In other words, attaining a unique optimal solution for which all OFs are simultaneously optimized is practically impossible. Accordingly, Pareto-optimal sets are considered non-dominated and satisfactory solutions in such cases, solutions in which amelioration at any OF is impossible unless at least one of OFs is debilitated [15]. The $\varepsilon$-constraint method is the most broadly employed a posteriori technique to cope with multiple OFs in relevant studies. This method permits outlining the Pareto front by acquiring efficient solutions and has proven achievements in the multiobjective RESCND problem in disturbances and operational fluctuations [30]. A significant advantage of this technique is that identical units and scales for OFs are not necessary for the solution process [6]. A multiobjective SCND problem is generally formulated as follows:

$$
\begin{aligned}
& \operatorname{Min} f_{1}, \ldots, f_{d}, \ldots, f_{j-1} \\
& \operatorname{Max} f_{j}, \ldots, f_{M} \\
& \text { s.t. } x \in \Omega
\end{aligned}
$$

where $\Omega$ represents the feasible solutions created through the intersection of constraints, $x$ denotes the variable vectors, and $f_{m}: \forall m=1,2, \ldots, M$ corresponds to the considered OFs optimized by altering $\varepsilon$ bounds in each execution iteration of the $\varepsilon$-constraint method. Moreover, $\varepsilon$ and $f_{g}$ indicate the $\varepsilon$ bound vector and the most important OF, respectively. The compact formulation of transforming the model mentioned above into an equivalent single-objective formulation via the $\varepsilon$-constraint method is as follows.

$$
\begin{aligned}
\operatorname{Min} & f_{g} \\
& f_{m} \leq \varepsilon_{m} ; \quad \forall m=1,2,3, \ldots, g-1, g+1, \ldots, M \\
& X \in \Omega .
\end{aligned}
$$

The Pareto front can be achieved by altering the value of the $\varepsilon$ vector and optimizing the equivalent singleobjective model for execution iterations of the mentioned technique. Accordingly, the determination of values for the $\varepsilon$ vector has a remarkable impact on the performance of the method to deal with multiple OFs, and this is the reason for developing the augmented $\varepsilon$-constraint method with proven advantages. Based on the augmented $\varepsilon$-constraint method, first, the pay-off table must be driven by independently optimizing the appended OFs in constraints as single-objective formulations to obtain proper values for the $\varepsilon$ bound vectors and determine the 
range of each $\varepsilon$. Therefore, each attained range is divided into $n_{m}$ equal intervals following equation (4.3) [26]. Here, $k$ corresponds to the execution iteration of the augmented $\varepsilon$-constraint approach.

$$
\text { Range }_{m}=r_{m}=f_{m}^{\text {Max }}-f_{m}^{\text {Min }} ; \quad \varepsilon_{m}^{k}=f_{m}^{\max }-\frac{r_{m}}{n_{m}} \times k ; \quad \forall m \neq g, \forall k=1, \ldots, n_{m}-1 .
$$

Since the primary form of the $\varepsilon$-constraint method cannot provide a compromise solution for each $\varepsilon$ vector, the mentioned basic formulation of the $\varepsilon$-constraint method should be revised, leading to the formation of the augmented method as follows [23].

$$
\begin{array}{ll}
\operatorname{Min} & \left(f_{g}(x)-\delta \times\left(\vartheta_{1} \frac{s_{1}}{r_{1}}+\vartheta_{2} \frac{s_{2}}{r_{2}}+\ldots+\vartheta_{g-1} \frac{s_{g-1}}{r_{g-1}}+\vartheta_{g+1} \frac{s_{g+1}}{r_{g+1}}+\ldots+\vartheta_{M} \frac{s_{M}}{r_{M}}\right)\right) \\
\text { s.t. } & \\
& f_{m}(x)+s_{m}=\varepsilon_{m} ; \quad \forall m=1,2,3, \ldots, g-1, g+1, \ldots, M \\
& s_{i} \in R^{+} \\
& x \in \Omega .
\end{array}
$$

In the formulation (4.4), $\vartheta_{m}$ corresponds to the priority of OF $m$ (where $\sum_{m \in M} \vartheta_{m}=1$ ); $s_{m}$ represents the positive auxiliary variable for standardizing the $\varepsilon$-constraint corresponding to OF $m$; and $\delta$ denotes a small number set to a value in the interval of $\left[10^{-6}, 10^{-3}\right]$. Besides, the term $\vartheta_{m} \frac{s_{m}}{r_{m}}$ ensures the provision of only one compromise solution of each $\varepsilon$ vector.

\subsection{Stochastic RO approach}

Here, a stochastic RO approach is applied to capture operational risks in the concerned SC. RO techniques are efficient methods to counter risks arising from inadequate historical data or the lack of knowledge for estimating the probability distribution of uncertain parameters [3]. Bertsimas and Sim [2] and Mulvey et al. [25] are the pioneers of RO methods. Notably, they proposed some of the most widely used methods in SCND under uncertainty. Mulvey et al. [25] proposed the RO technique considering the variance as an index for measuring the variability of OFs in scenario-based stochastic programming problems. The proposed methodology has demonstrated success in RO programming and the relevant literature. Mulvey et al. [25] proposed two measures, including solution robustness (SR) and model robustness (MR). The SR indicator tries to achieve a solution very close to the optimal one, and the MR measure ensures the feasibility of the solution by considering a unique function for the penalty.

The general compact formulation of applying the Mulvey RO method in SCND under uncertainty is as follows.

$$
\begin{aligned}
& \text { Min } \mathrm{TC}=\sum_{s \in S} \pi_{s} Z_{s}+\lambda \sum_{s \in S} \pi_{s}\left(Z_{s}-\sum_{s^{\prime} \in S} \pi_{s^{\prime}} Z_{s^{\prime}}\right)^{2}+\xi\left(\sum_{s \in S} \pi_{s} \delta_{s}^{2}\right) \\
& \text { s.t. } \\
& A x=b \\
& B_{s} x+C_{s} y_{s}+\delta_{s}=e_{s} \\
& s \in S \\
& y_{s} \geq 0 \\
& s \in S \\
& x \geq 0 \text {. }
\end{aligned}
$$

Due to the non-linear terms in the OF (4.5), the complexity of the proposed robust formulation intensely increases and cannot be applied to large-scale problems. To avoid the non-linearity condition, Leung et al. [21] proposed an equivalent linear formulation for the Mulvey RO method as follows.

$$
\operatorname{Min} Z=\sum_{s \in S} \pi_{s} z_{s}+\lambda \sum_{s \in S} \pi_{s}\left[\left(z_{s}-\sum_{s^{\prime} \in S} \pi_{s^{\prime}} z_{s^{\prime}}\right)+2 \tau_{s}\right]+\xi \sum_{s \in S} \pi_{s} \delta_{s}
$$


s.t.

$$
\begin{array}{lc}
A x=b & \\
B_{s} x+C_{s} y_{s}+\delta_{s}=e_{s} & s \in S \\
z_{s}-\sum_{s \in \Omega} \pi_{s} z_{s}+\tau_{s} \geq 0 & s \in S \\
y_{s} \geq 0 & s \in S \\
x \geq 0 & \\
\tau_{s} \geq 0 & s \in S .
\end{array}
$$

Note that $\lambda$ and $\xi$ represent parameters, including variability weight (VW) and risk aversion weight (RAW). Besides, $\xi_{s}$ and $\delta_{s}$ indicate auxiliary variables employed in the linearization of the proposed robust formulation. The robust OF (4.5) consists of three terms, where the first and second terms indicate the mean and variance of the concerned OF under disruption scenarios, respectively. Elevating the VW reduces the sensitivity of the model to the fluctuations in input parameters. These two terms guarantee the robustness of the attained solution. Furthermore, the third term measures the model in the presence of infeasibility, which underlies the robust formulation. The optimal robust solution should be located in the feasible region of the problem and in the proximity of optimality. Readers can refer to $[21,25]$ for more detailed information about the Mulvey optimization approach.

\section{IMPLEMENTATION AND EVALUATION}

\subsection{Case study}

A case study of the steel industry in Iran is considered to ensure the applicability of the proposed formulation. Steel-based products include various types of ingots, iron sheets, rebars, iron angles, pipes, and beams widely used in many industries. The primary stimulation that drove the steel industry to redesign its SC network is as follows: Given the alternation in government policies towards polluting industries, SCs must strive to reduce the environmental impact of their activities. Iran's historical background in the emergence of numerous threats, e.g., exchange rate fluctuations, suppliers' non-commitment, international sanctions, earthquakes, and floods, indicates that the steel industry needs thorough planning to deal with operational and disruption risks. Moreover, reducing the cost of purchasing raw materials by extracting them from recyclable scrap iron is the main criterion for evaluating the redesign of the steel SC network.

Herein, three real datasets have been used to evaluate the performance and accuracy of the proposed model. The mentioned datasets are collected based on optimistic, most likely, and pessimistic scenarios, respectively. The optimistic approach or scenario means considering fewer candidate facilities, fewer establishment or unit costs, and fewer unit environmental impacts. Besides, more capacity for facilities, less product demand, less capacity reduction due to disruptions, fewer periods, fewer disruptions, fewer available routes, less disrupted routes, and fewer available backup suppliers are considered in the optimistic scenario. The number of product families, raw materials, available landfills, and fortification levels are the same for all three mentioned scenarios. Table 1 lists the specifications of the applied datasets associated with the case study.

Steel products comprise six families of semi-finished steel products, hot-rolled products, cold-rolled products, steel sections, tubes and profiles, and stainless steel; thus, six products are considered in the case study. Each product family consists of various products (Tab. 2). Five raw materials are required for manufacturing steel products, namely iron ore, coal, limestone, scrapped iron, and alloy elements.

Three technologies, including blast furnace, basic oxygen furnace, and electric arc furnace, can be used to combine various raw materials under molten circumstances to make steel alloys with different physical and chemical properties.

Based on the most likely dataset, the Iranian steel industry's SC simultaneously serves 10 primary market zones across the country. There are also 10 primary suppliers or mines of iron ore and other alloying elements 
TABLE 1. Specifications of considered datasets.

\begin{tabular}{lllllllllllll}
\hline \hline \multirow{2}{*}{ Datasets } & \multicolumn{10}{c}{ Indices } \\
\cline { 2 - 12 } & $|B|$ & $|C|$ & $|D|$ & $|I|$ & $|L|$ & $|M|$ & $|P|$ & $|S|$ & $|W|$ & $|R|$ & $|O|$ & $|T|$ \\
\hline 1 (Optimistic) & 3 & 10 & 6 & 5 & 7 & 9 & 6 & 8 & 4 & 4 & 5 & 6 \\
2 (Most likely) & 5 & 10 & 8 & 5 & 7 & 13 & 6 & 10 & 5 & 6 & 7 & 8 \\
3 (Pessimistic) & 8 & 10 & 9 & 5 & 7 & 15 & 6 & 12 & 7 & 9 & 9 & 9 \\
\hline
\end{tabular}

TABLE 2. Steel products families and associated sub-products.

\begin{tabular}{ll}
\hline \hline Product family & Sub-products \\
\hline Semi-finished steel products & $\begin{array}{l}\text { Steel ingots (billet, bloom, round ingots), slab, direct reduced iron } \\
\text { (DRI), pellet, and briquette }\end{array}$ \\
Hot rolled products & $\begin{array}{l}\text { Hot-rolled coil, hot sheet, and plate, acid-washed sheet, chequer plate, } \\
\text { carbon steel sheet } \\
\text { Cold-rolled sheet (oily), galvanized sheet, colored sheets, tin-coated } \\
\text { sheet, Galvano sheet (allogenic), aluminized sheet, POSMAC steel }\end{array}$ \\
Cold-rolled products & HEA beam, IPE beam, I\&C studs, rebar, wire rope, steel wire, steel \\
angel, flat bar, corner iron, tubes, and profiles & Seamed pipe, seamless pipe, spiral tube, steel profile, precision tube \\
Steel sections & $\begin{array}{l}\text { Stainless coil, stainless plate and sheet, stainless rebar, stainless pipe, } \\
\text { stainless profile }\end{array}$ \\
stainless steel &
\end{tabular}

related to steel that provide raw materials for production. There are also five ready-to-serve backup suppliers to deal with disruptions and operational risks that can be selected at the strategic decision-making level. In this case study, five candidate facilities for warehouses, 13 candidate facilities for production centers, and eight candidate facilities for distribution centers are taken into account to establish new required facilities.

Figure 2 demonstrates the locations of various candidates and existing facilities involved in the Iran steel industry's SC based on the most likely scenario.

Assuming that the existing industries in all provinces of Iran are the primary markets of steel products, there are 31 target markets. Given the geographical neighborhood of provinces, market zones are downsized to 10 market clusters to alleviate model complexity. Table 3 illustrates the provinces placed in each market cluster based on the neighboring criterion.

Table 4 indicates the estimated average demands for steel product families in first markets for every six months. Note that product demand varies from period to period and under each disturbance.

Tables 5-7 provide information on the average capacity of candidate facilities and backup suppliers under disruption, taking into account the expected value (EV) of estimated capacities based on optimistic, pessimistic, and most likely scenarios, respectively.

\subsection{Computational results and managerial insights}

The proposed model is implemented based on three datasets associated with a case study of the steel industry to demonstrate the effectiveness and validity of the investigated robust formulation. Datasets 1 to 3 correspond to optimistic, most likely, and pessimistic scenarios, respectively. The model is compiled in GAMS 24.2.1 and solved using the CPLEX package. It is also executed on an ASUS laptop with a $2.3 \mathrm{GHz}$ Intel Core i7 $3610 \mathrm{QM}$ and 8 GB RAM DDR3 under 64-bit Windows 10.

Some measures are necessary to evaluate the performance and reliability of the presented model. Technically speaking, sensitivity analysis should be implemented to assess the impact of particular parameters on OFs 


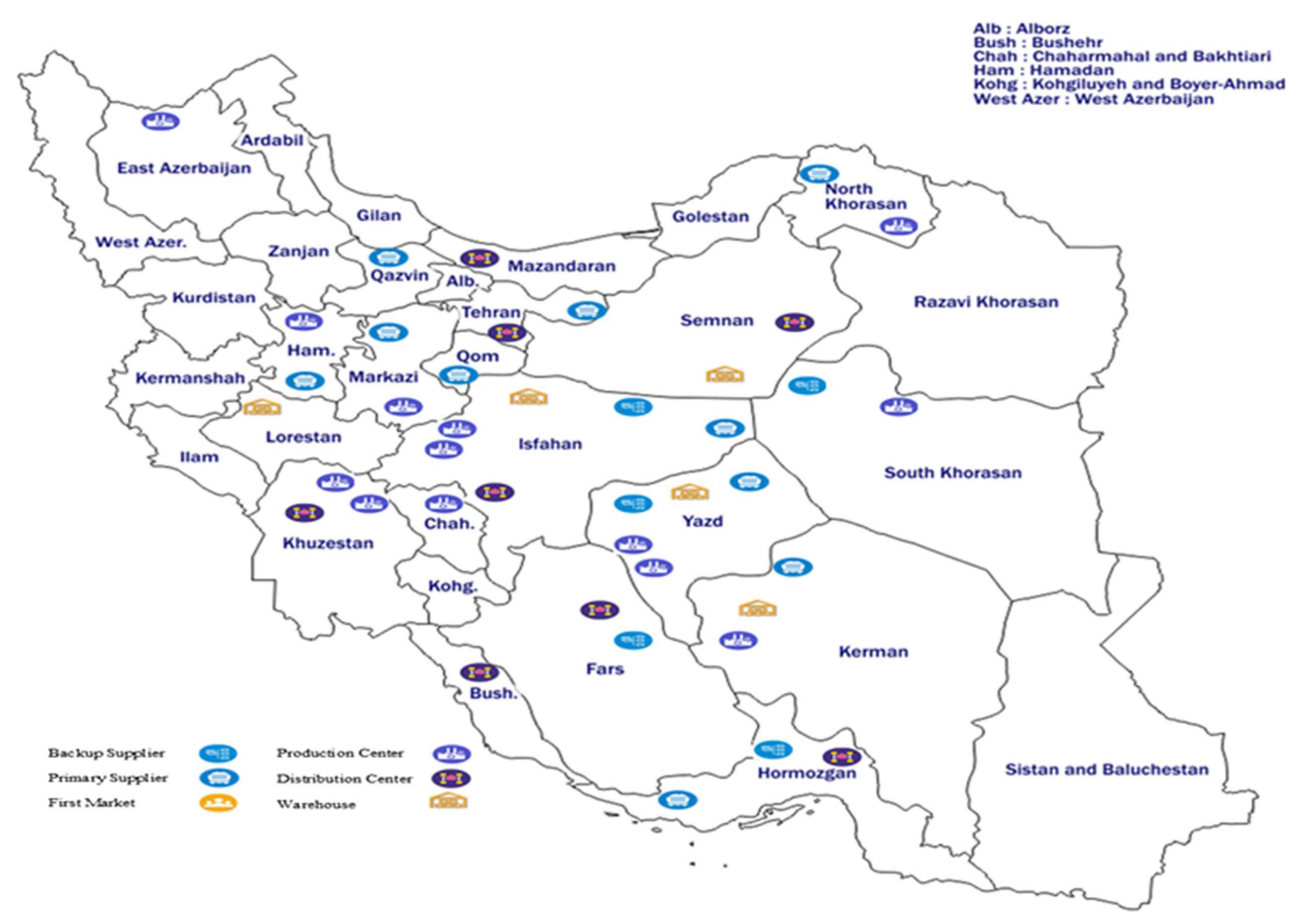

FIGURE 2. Locations of the candidate and existing facilities involved in the Iran steel industry's $\mathrm{SC}$ based on the most likely scenario.

TABle 3. Provinces considered in each market cluster based on geographical criteria.

\begin{tabular}{ll}
\hline \hline Market cluster & Provinces in each cluster \\
\hline 1 & Tehran, Alborz, Qazvin \\
2 & Qom, Markazi, Isfahan \\
3 & Semnan, Golestan, Mazandaran \\
4 & North, Razavi, and South Khorasan \\
5 & Gilan, Ardabil, East Azerbaijan \\
6 & West Azerbaijan, Zanjan, Kurdistan \\
7 & Hamadan, Lorestan, Ilam, Kermanshah \\
8 & Khuzestan, Chaharmahal and Bakhtiari \\
9 & Yazd, Fars, Bushehr \\
10 & Sistan and Balouchestan, Kerman, Hormozgan \\
\hline
\end{tabular}

and investigate the conflicts between them. As this study developed a robust and RESCND problem under operational risks and disturbances, the achievements of the proposed robust and resilient formulation must be investigated.

RESCND involves both pre-event and post-event decisions. The pre-event phase incorporates scenarioindependent or strategic decisions such as establishing facilities and determining the criticality of nodes. Furthermore, the post-event phase involves scenario-dependent or operational decisions such as specifying the flow between facilities, inventory level at a particular facility, the number of lost sales at first markets, and decisions associated with the pre-positioned EI. 
TABLE 4. Average demands for steel products families in first markets for every six months or one period (Million ton).

\begin{tabular}{lllllll}
\hline \hline \multirow{2}{*}{$\begin{array}{l}\text { Primary } \\
\text { market }\end{array}$} & $\begin{array}{l}\text { Semi-finished } \\
\text { steel products }\end{array}$ & $\begin{array}{l}\text { Hot rolled } \\
\text { products }\end{array}$ & $\begin{array}{l}\text { Cold-rolled } \\
\text { products }\end{array}$ & $\begin{array}{l}\text { Steel } \\
\text { sections }\end{array}$ & $\begin{array}{l}\text { Tubes and } \\
\text { profiles }\end{array}$ & $\begin{array}{l}\text { Stainless } \\
\text { steel }\end{array}$ \\
\hline 1 & 1.5 & 0.71 & 0.46 & 1.28 & 0.76 & 0.94 \\
2 & 2.6 & 1.11 & 0.42 & 1.03 & 0.58 & 0.99 \\
3 & 2.2 & 1.13 & 0.72 & 1.27 & 0.93 & 1.01 \\
4 & 2 & 1.22 & 0.62 & 1.09 & 0.67 & 1.09 \\
5 & 1.8 & 0.78 & 0.63 & 0.86 & 0.41 & 0.73 \\
6 & 3.1 & 0.85 & 0.6 & 0.8 & 0.93 & 0.62 \\
7 & 2.3 & 0.7 & 0.65 & 0.98 & 0.94 & 0.84 \\
8 & 3.9 & 0.89 & 0.52 & 0.87 & 0.75 & 1.05 \\
9 & 2.5 & 0.91 & 0.62 & 1.38 & 0.52 & 1.09 \\
10 & 2.8 & 0.87 & 0.66 & 1.23 & 0.41 & 1.08 \\
Total & 24.7 & 9.17 & 5.9 & 10.79 & 6.9 & 9.44 \\
demands & & & & & & \\
\hline
\end{tabular}

TABLE 5. Average capacities of candidate warehouses for raw materials in a period (Million tons).

\begin{tabular}{|c|c|c|c|c|c|c|c|c|c|c|}
\hline \multirow[b]{3}{*}{ Warehouses } & \multicolumn{10}{|c|}{ Raw materials } \\
\hline & \multicolumn{5}{|c|}{ Holding capacity } & \multicolumn{5}{|c|}{ Supply capacity } \\
\hline & $\begin{array}{l}\text { Iron } \\
\text { ore }\end{array}$ & Coal & Limestone & $\begin{array}{l}\text { Scrapped } \\
\text { iron }\end{array}$ & $\begin{array}{l}\text { Alloy } \\
\text { elements }\end{array}$ & $\begin{array}{l}\text { Iron } \\
\text { ore }\end{array}$ & Coal & Limestone & $\begin{array}{l}\text { Scrapped } \\
\text { iron }\end{array}$ & $\begin{array}{l}\text { Alloy } \\
\text { elements }\end{array}$ \\
\hline Isfahan & 161 & 29 & 17 & 89 & 4 & 33 & 8 & 5 & 19 & 3 \\
\hline Kerman & 145 & 22 & 23 & 82 & 8 & 32 & 8 & 5 & 16 & 3 \\
\hline Lorestan & 155 & 23 & 22 & 88 & 4 & 35 & 8 & 6 & 12 & 3 \\
\hline Semnan & 138 & 30 & 23 & 66 & 7 & 41 & 10 & 5 & 12 & 3 \\
\hline Yazd & 129 & 34 & 16 & 69 & 6 & 35 & 8 & 3 & 14 & 1 \\
\hline
\end{tabular}

Table 8 presents the strategic or pre-event decisions of the SC and the calculated values of OFs in each iteration of the augmented $\varepsilon$-constraint method. The insights reveal that considering some resilience strategies such as establishing fewer facilities and transportation links and minimizing the total flows to the established facilities significantly decrease the TNRN but increase system costs.

\subsubsection{Assessing the conflict between OFs}

A problem can be formulated as multi-objective only in the case of conflicts between OFs. Based on Section 4.1, optimal solutions can be attained by solving the equivalent single-objective formulation generated by the augmented $\varepsilon$-constraint method. The mentioned formulation is generated by varying $\varepsilon$ bounds in each execution iteration of the applied method. If a Pareto front is plotted based on the pay-off between two pairs of OFs and the achieved plot behaves strictly decreasing or increasing, the OFs are considered conflicting. This research aims to simultaneously minimize the TC and TEI of the SC and the TNRN associated with the network, so the conflict between these three OFs should be evaluated. Figures 3a-3c illustrates the conflict and pay-off between OFs for optimistic, most likely, and pessimistic execution scenarios, respectively.

Based on the observations, an increment in TC remarkably decreases the TNRN, and the corresponding plot behaves strictly decreasing, so the investigated OFs are conflicted. Such a behavior is reasonably expected, as an effort to mitigate the non-resiliency of the system or enhance resilience capabilities would be costly. Given 
TABLE 6. Average capacities of candidate production centers for products in a period of every six months (Million tons).

\begin{tabular}{lllllll}
\hline \hline & \multicolumn{5}{c}{ Product families } \\
\cline { 2 - 6 } Production centers & $\begin{array}{l}\text { Semi-finished } \\
\text { steel products }\end{array}$ & $\begin{array}{l}\text { Hot rolled } \\
\text { products }\end{array}$ & $\begin{array}{l}\text { Cold-rolled } \\
\text { products }\end{array}$ & $\begin{array}{l}\text { Steel } \\
\text { sections }\end{array}$ & $\begin{array}{l}\text { Tubes and } \\
\text { profiles }\end{array}$ & $\begin{array}{l}\text { Stainless } \\
\text { steel }\end{array}$ \\
\hline Chaharmahal and & 0 & 5.69 & 1.12 & 1.77 & 1.92 & 1.07 \\
Bakhtiari & & & & & \\
East Azerbayjan & 5.95 & 1.79 & 1.27 & 1.89 & 1.52 & 0.99 \\
Hamadan & 5.08 & 0 & 0.93 & 1.64 & 0 & 1.95 \\
Isfahan (north) & 4.65 & 4.23 & 0 & 0 & 0.95 & 1.67 \\
Isfahan (south) & 9.9 & 4.67 & 1.35 & 0 & 1.76 & 2.02 \\
Kerman & 0 & 5.35 & 1.54 & 1.4 & 1.77 & 0.92 \\
Khouzestan & 10.08 & 3.3 & 0 & 0.83 & 0 & 1.09 \\
(north) & & & & & 0 & 2 \\
Khouzestan & 0 & 4.23 & 1.24 & 1.1 & 0 & 1.52 \\
(south) & & & & & & 0.94 \\
Markazi & 5.64 & 0 & 0.94 & 0 & 1.74 & 1.27 \\
North Khorasan & 10.27 & 1.97 & 1.22 & 0.97 & 0 & 0.91 \\
South Khorasan & 10.11 & 4.55 & 1.65 & 1 & 1.07 & 1.19 \\
Yazd (north) & 0 & 3.43 & 0 & 1.3 & 0 & 1.36 \\
Yazd (sourth) & 4.3 & 0 & 1.58 & 1.89 & & \\
\hline
\end{tabular}

TABLE 7. Average available capacities of backup suppliers for raw materials in a period (Million tons).

\begin{tabular}{llllll}
\hline \hline & \multicolumn{5}{c}{ Raw material } \\
\cline { 2 - 6 } Backup suppliers & Iron ore & Coal & Limestone & Scrapped iron & Alloy elements \\
\hline Fars & 30.9 & 13.9 & 12.2 & 17.7 & 6.9 \\
Hormozgan & 32.1 & 12.5 & 12.6 & 16.1 & 6.8 \\
Isfahan & 35.8 & 12 & 12.8 & 13.9 & 10.2 \\
South Khorasan & 25 & 13.7 & 8.5 & 15.9 & 5.9 \\
Yazd & 28.5 & 10.7 & 9 & 17.9 & 5.3 \\
\hline
\end{tabular}

the OFs of minimization, the separate conflict of TEI against TC and TNRN is proved similarly. Based on these insights, TC, TEI, and TNRN as OFs are conflicted, and the problem can be formulated as a multi-objective model.

\subsubsection{The cost-efficiency of the applied single and multiple resilience strategies}

Herein, contingency and precautionary resilience measures, including holding pre-positioned emergency inventory (HPEI), multiple sourcing (MS), taking advantage of lateral transshipment (LT), fortification of facilities (FF), and providing backup facilities (PBF) are considered simultaneously. Besides, non-resiliency measures such as $\mathrm{NC}, \mathrm{FC}$, and $\mathrm{NCr}$ are employed to develop an $\mathrm{SC}$ with a resilient network. This section evaluates the effect of executing the mentioned strategies independently on the developed model's cost performance. To this end, the presented model is implemented given three random datasets associated with a benchmarked example, and the corresponding results are extracted and analyzed.

Figure 4 demonstrates the impacts of applying independent resilience strategies on the TC. Evidently, implementing structural strategies, including MS, HPEI, FF, PBF, and LT, leads to a remarkable cost change. In contrast, the network resiliency measures including $\mathrm{FC}, \mathrm{NC}$, and $\mathrm{NCr}$, sharply increase the TC. There is a 
TABLE 8. Taken pre-event decisions of the investigated SC and the calculated values of OFs.

\begin{tabular}{|c|c|c|c|c|c|c|c|c|}
\hline \multicolumn{9}{|c|}{ Optimistic scenario (Dataset1) } \\
\hline \multirow{2}{*}{$\begin{array}{l}\text { Iteration of } \\
\varepsilon \text {-constraint }\end{array}$} & \multicolumn{3}{|c|}{ Pay-off between OFs } & \multicolumn{5}{|c|}{ Location of SC nodes } \\
\hline & $\mathrm{TC}$ & TEI & TNRN & $\mathrm{BS}$ & WH & $\mathrm{PC}$ & $\mathrm{DC}$ & $\mathrm{CC}$ \\
\hline 1 & 710 & 620 & 425 & 1,3 & $1,2,4$ & $1,3,4,6,9$ & $2,4,5$ & $2,4,5,7$ \\
\hline 2 & 805 & 544 & 392 & 1,3 & 1,4 & $1,3,4,6,8$ & $2,3,4$ & $2,3,5,6$ \\
\hline 3 & 915 & 372 & 312 & 2,4 & $1,3,4$ & $2,3,5,7,8$ & $2,3,5$ & $1,4,5,8$ \\
\hline 4 & 1040 & 291 & 233 & 2,3 & $2,3,4$ & $2,3,4,5,8$ & $1,2,5$ & $1,4,7,8$ \\
\hline 5 & 1220 & 259 & 180 & 1,4 & 2,3 & $2,4,6,7,9$ & $2,3,5$ & $1,3,5,9$ \\
\hline 6 & 1283 & 146 & 162 & 1,4 & 2,3 & $1,3,4,5,9$ & $1,4,5$ & $1,2,4,5$ \\
\hline \multicolumn{9}{|c|}{ Most likely scenario (Dataset2) } \\
\hline 1 & 1270 & 1100 & 625 & $1,4,6$ & $1,2,4,5$ & $2,3,6,7,9,11$ & $1,4,6,7,8$ & $1,4,6,7,9$ \\
\hline 2 & 1322 & 872 & 454 & $1,3,6$ & $1,2,3,5$ & $2,4,5,9,11,12$ & $1,4,6,7,8$ & $1,2,5,7,9$ \\
\hline 3 & 1431 & 688 & 310 & $2,4,5$ & $2,3,5$ & $1,2,4,5,6,9,13$ & $1,3,6,7,8$ & $2,3,6,8,9$ \\
\hline 4 & 1850 & 569 & 277 & $1,3,4$ & $2,3,4,5$ & $1,3,5,8,11,13$ & $2,3,4,6,7$ & $1,4,5,8,9$ \\
\hline 5 & 2466 & 361 & 190 & $1,4,5$ & $1,3,4,5$ & $2,3,6,8,11,12$ & $2,3,4,6,7$ & $1,2,5,8,9$ \\
\hline 6 & 3121 & 288 & 126 & $2,4,5$ & $1,2,4,5$ & $2,3,6,7,10,13$ & $2,3,5,6$ & $2,3,7,8,9$ \\
\hline \multicolumn{9}{|c|}{ Pessimistic scenario (Dataset3) } \\
\hline 1 & 1750 & 1350 & 977 & $1,3,4,6$ & $2,4,6,7$ & $1,3,6,7,12,13,15$ & $2,4,6,7$ & $2,3,5,6,7,8$ \\
\hline 2 & 1995 & 1210 & 673 & $2,3,5,6$ & $1,4,5,7$ & $2,3,4,6,9,13,15$ & $2,4,5,6$ & $1,4,5,6,7,8$ \\
\hline 3 & 2242 & 952 & 590 & $2,3,4,6$ & $2,4,5,6,7$ & $1,4,6,8,11,12,15$ & $1,3,5,6$ & $2,3,5,6,8$ \\
\hline 4 & 2773 & 814 & 374 & $1,2,5,7$ & $1,3,5,6,7$ & $2,5,6,7,10,11,14$ & $2,4,5,7$ & $2,3,5,6,8$ \\
\hline 5 & 3450 & 542 & 263 & $1,3,5,6$ & $2,3,4,6,8$ & $2,3,4,7,11,12,13$ & $2,3,4,6$ & $1,3,5,6,8$ \\
\hline 6 & 4933 & 417 & 193 & $2,3,5,7$ & $1,5,6,8$ & $1,2,4,6,7,8,11$ & $1,2,4,6$ & $1,2,5,6,8$ \\
\hline
\end{tabular}

(a)

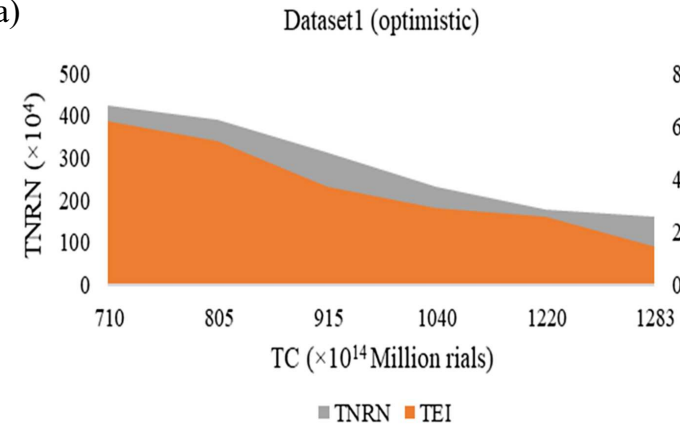

(b)

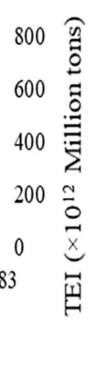

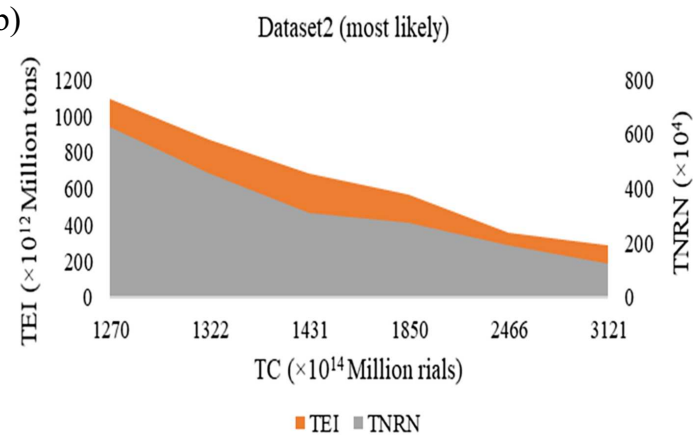

(c)

Dataset3 (pessimistic)

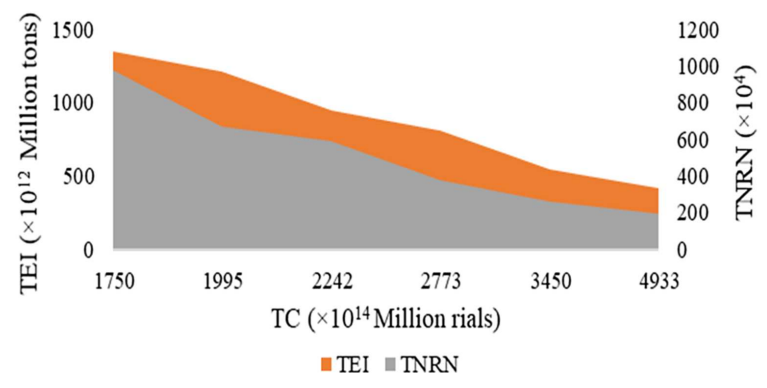

Figure 3. (a) The conflict between OFs for dataset1. (b) The conflict between OFs for dataset2.

(c) The conflict between OFs for dataset3. 


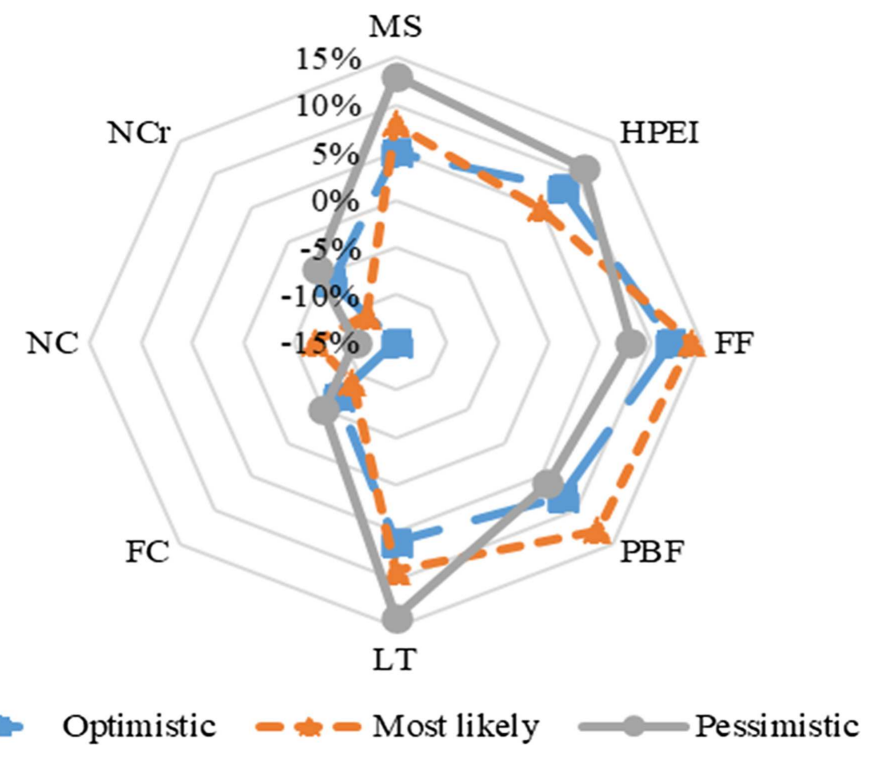

FiguRE 4. The cost-efficiency of utilizing independent structural and network resiliency measures on the TC.

logic behind this behavior; considering independent structural strategies will increase the products' flow, the facilities' capacity, and the nodes' ability to store or purchase EI. All the mentioned measures will significantly decrease the cost of lost sales in CZs and the cost of inventory at facilities, leading to an overall reduction in the TC. On the other hand, implementing network resiliency measures, including FC, NC, and $\mathrm{NCr}$ will minimize the TNRN that does not come free and requires a rise in TC that leads to negative cost performances.

Figure 5 illustrates the cost performance of applying multiple resilience mitigation and preventive strategies on the SC in disruptions. Evidently, employing multiple structural strategies, including MS, HPEI, FF, PBF, and LT, reduces costs. Applying some more structural strategies decreases the risk exposure costs and, consequently, the TC. This behavior is reasonable because adopting more structural strategies provides more customer satisfaction via decreasing product shortages in markets and making SC fortified in the incidence of disturbances that reduce the TC. Since TNRN can be decreased only by reducing the associated measures, including $\mathrm{FC}, \mathrm{NC}$, and $\mathrm{NCr}$, it costs much; therefore, considering network resilience strategies and structural resilience strategies remarkably reduces the cost performance.

\subsubsection{Determining the impact of the operational capacity of facilities}

The behavior of the developed model should be assessed under changing particular parameters to guarantee model validation. Only the impact of uncontrollable parameters influenced by external factors can be evaluated. To this end, operational capacities, including the delivery, holding, and manufacturing capacity that are respectively associated with distribution centers, warehouses, and production centers, are considered.

Figure 6 displays the simultaneous effects of changing the operational capacity of facilities on the TC and the TNRN of the SC. The results confirm that an increase in the operational capacities leads to a decline in the TC, but the TNRN will increase. The model behaves identically for all three random data sets. The logic behind this behavior can be described as follows: Enhancing the operational capacity of facilities leads to manufacturing, holding, and distribution of more products, which increases the input and output flow to different nodes. Besides, this will significantly decrease lost sales and product inventories, thereby reducing the 


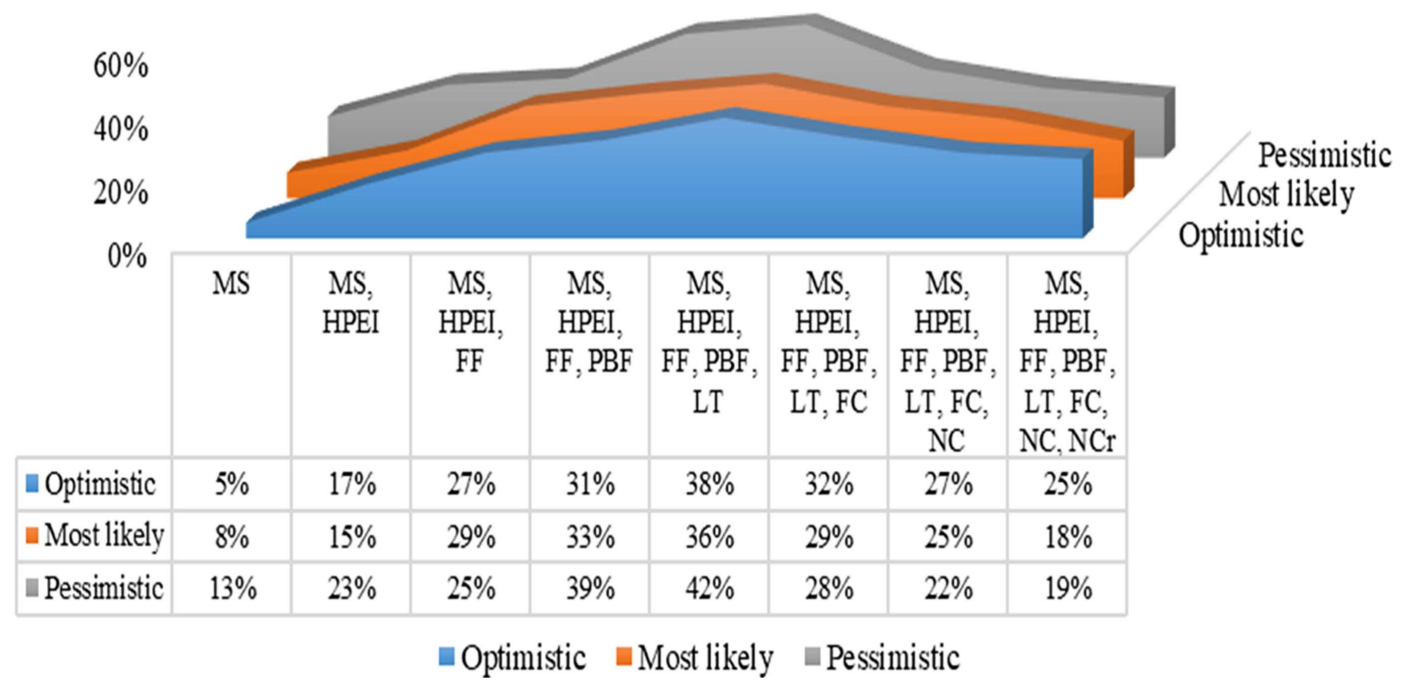

FIgURE 5. The efficiency of applied multiple resilience strategies in terms of cost.

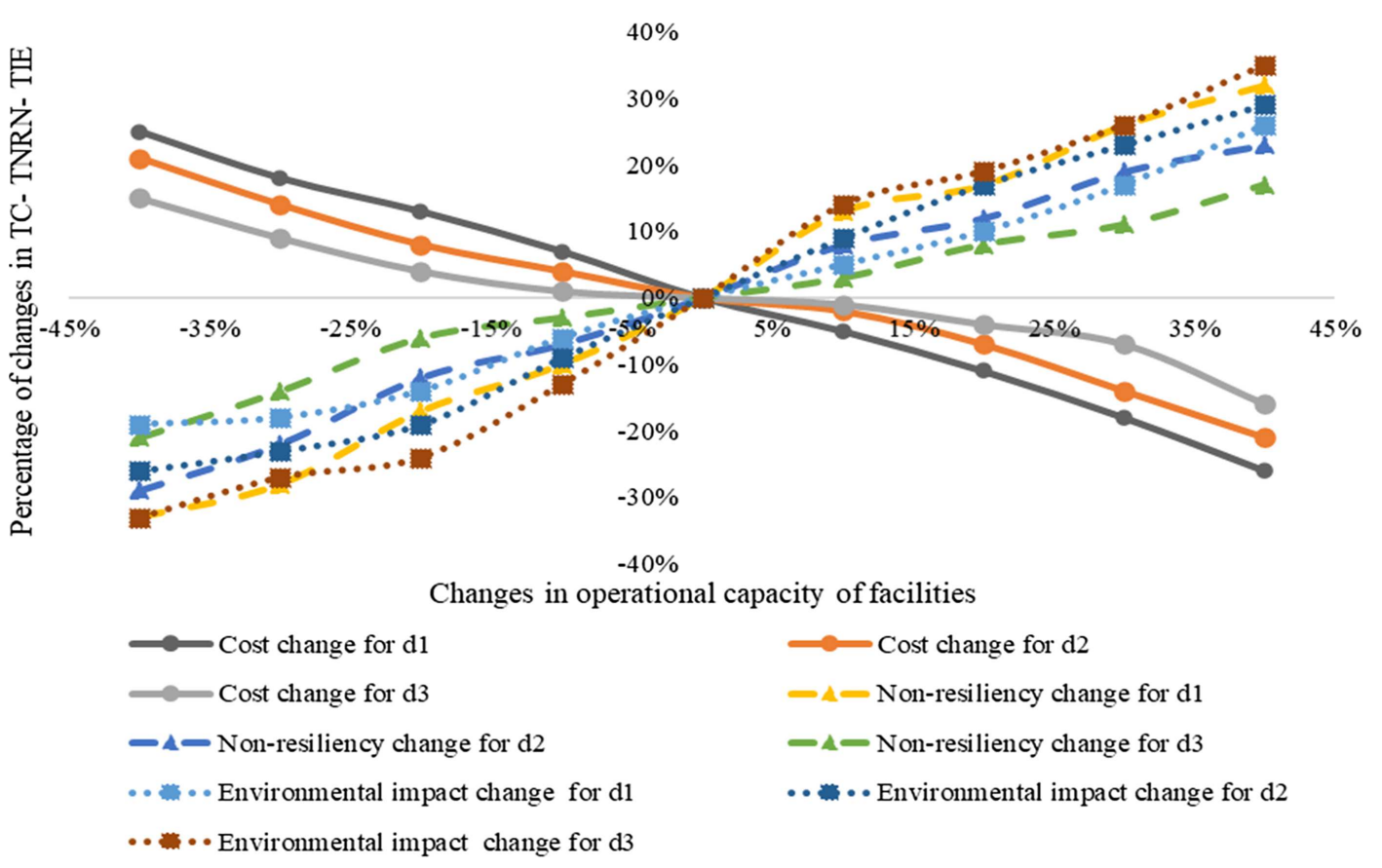

FIGURE 6. Impact of altering the operational capacity of facilities on TC and TNRN.

shortage and holding costs to decrease the TC. Furthermore, raising the number of established facilities, the amount of flow between different nodes, and the number of opened links will increase $\mathrm{NCr}, \mathrm{NC}$, and FC criteria, leading to a significant increment in the level of TNRN. Note that $\mathrm{d} 1$, d2, and $\mathrm{d} 3$ represent optimistic, most likely and pessimistic datasets. 


\subsubsection{The trade-off between SR and $M R$}

Choosing a proper RAW represented by $\xi$ is a significant point in the Mulvey RO method. This coefficient is employed to attain a trade-off between SR and MR. Depending on the conditions, DMs make choices in a risk-averse or risk-seeking manner. A risk-seeking DM attempts to minimize TC and is thus inclined to consider smaller values of $\xi$. Besides, a risk-averse DM tends to consider higher values of $\xi$ to prevent shortages and lost sales in the market zones. Therefore, varying RAW leads to a trade-off between demand under-fulfillment (DUF) and TC. Figure 7 illustrates the trade-off between MR and SR over various values of RAW for three random datasets. The analysis results are considered to determine a proper value for RAW in the proposed optimization model. The overall behavior indicates that an increment in the RAW increases the TC corresponding to SR. In other words, it decreases the DUF representing MR. Given the observed behavior, the more the RAW increases, the more the model tends to attain feasible solutions. Eventually, increasing the value of RAW decreases the DUF or MR to 0 . The results indicate identical behaviors for applying all datasets to the presented model. Notably, these findings are perfectly compatible with the outcomes achieved by Dehghani et al. [7] and Jabbarzadeh et al. [19].

Selecting a proper value for RAW is a significant point in the Mulvey RO method. Fortunately, the above analysis can attain this goal, and DM significantly prefers the feasible solutions obtained in this way. The impact of altering RAW on TC and DUF is analyzed for all three random datasets, and the proper values of $\xi$ for optimization models are determined by interpreting the results in Figure 7. Based on this analysis, the optimal value of $\xi$ is respectively considered 13,15 , and 16 for datasets 1 to 3 . The rationale behind this decision is explained as follows: The DUF is equal to zero, and TC does not vary for values of $\xi$ higher than 13,15 , and 16 for datasets 1 to 3, respectively, which means both MR and SR will be determined as stable and with a uniform amount. Note that DMs can consider different values for $\xi$ due to the problem specifications, DMs' aspiration, and business environment conditions.

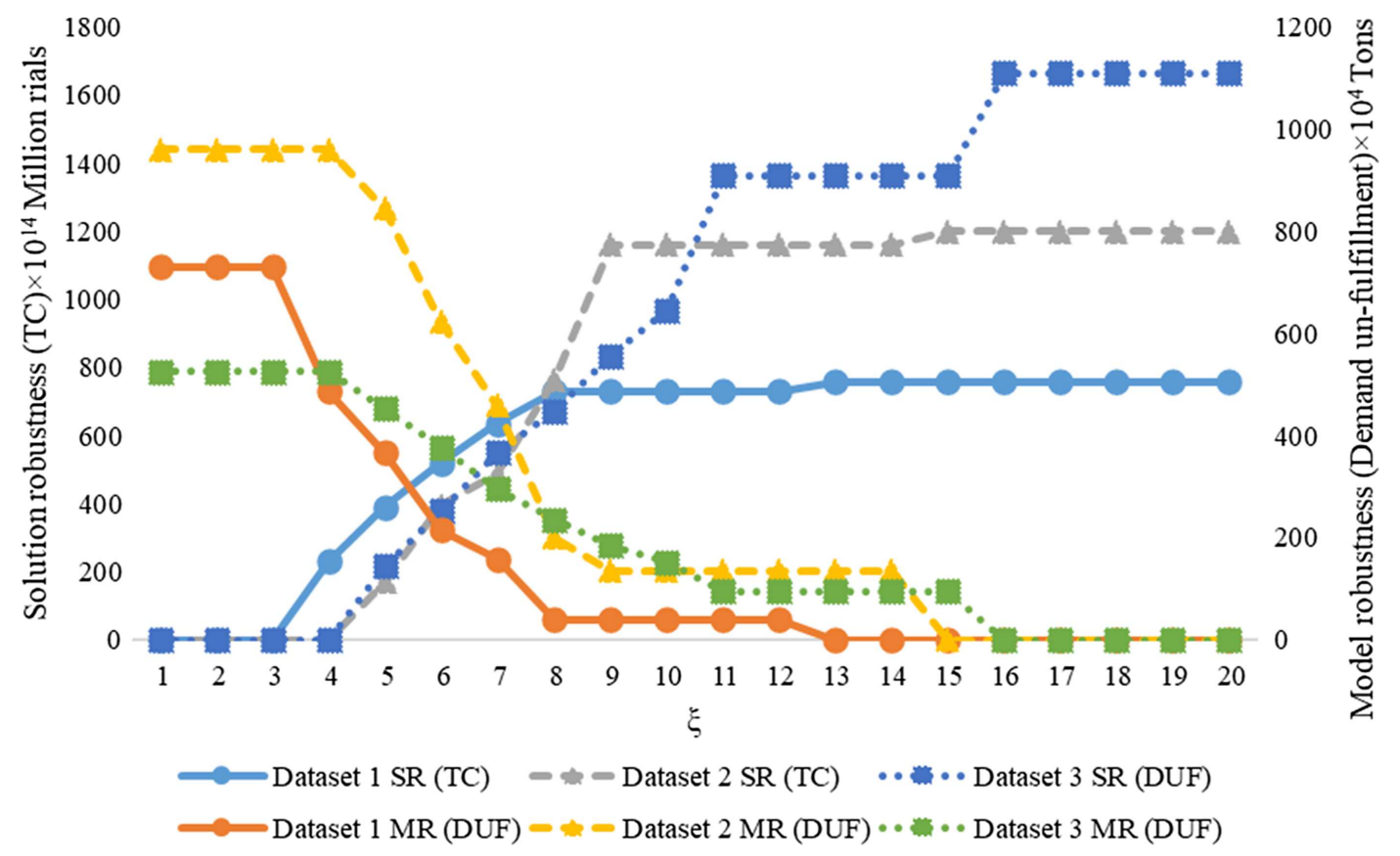

FigURE 7. Impact of varying RAW on TC and DUF for all three datasets. 


\subsubsection{The benefits of the robust formulation}

Appling RO to a deterministic model must provide achievements for the the SC stakeholders. In the case of operational risks, we consider two types of costs for the system, including exposure and prevention costs. The costs of risk exposure involve the losses that occur after risk occurrence. In contrast, prevention costs consist of creating readiness and flexibility in the SC under risks. Thus, this section investigates the applicability and cost-efficiency of the applied RO.

Figure 8 presents a comparison between the two-stage and SBR (Mulvey) methods from a performance perspective for three random datasets. Given the minimization of OFs, the robust model yields higher TC and TNRN in the short term because managing operational risks does not come free. On the other hand, the robust model can mitigate and prevent risks, which is a remarkable advantage. Applying RO to the model will decrease robustness and risk exposure costs by reducing lost sales and dealing with fluctuations in the long term. In this study, we have relied on the scenario-based RO model to make the required decisions, attain solutions, and analyze the results.

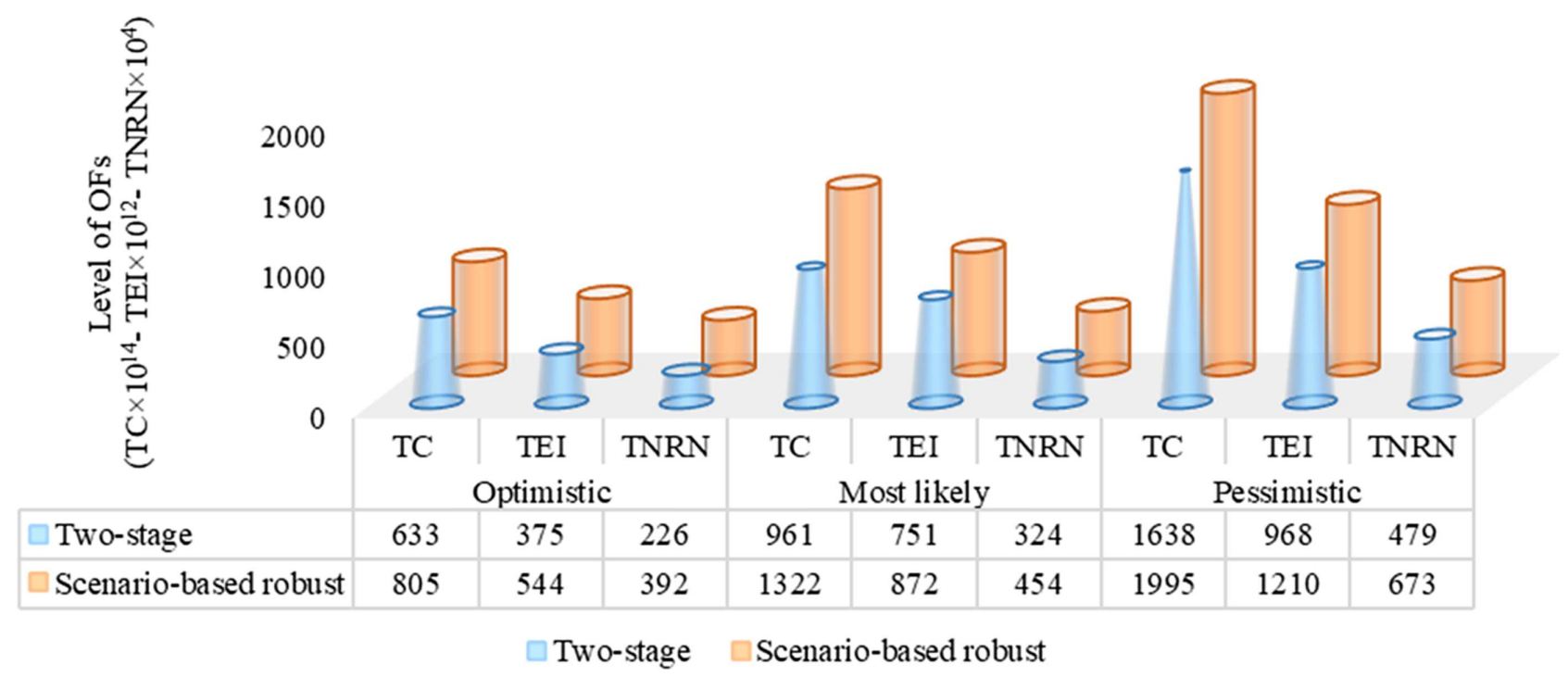

FiguRE 8. Comparing the performance of the two-stage and SBR formulation.

\section{Conclusion}

Organizations, especially manufacturing companies, are constantly exposed to different risks and damages due to operational risks and disruptions. System vulnerability is rooted in the instability of the business environment, natural disasters, human-made damages, and operational fluctuations. Therefore, a lack of sufficient knowledge of SC risk management can lead to irreparable consequences such as losses in financial performance, customer satisfaction, and brand credibility. Relevant research on RESCND has been dedicated to identifying potential disturbance scenarios, suggesting precautionary and contingency resilience measures, and applying efficient independent resilience strategies to tackle disruptions. The reviewed studies provide some insights; for instance, considering multiple resilience strategies, assuming complete disturbances in transportation links, investigating network non-resiliency criteria such as $\mathrm{NC}, \mathrm{NCr}$, and $\mathrm{FC}$, and simultaneously employing mitigation and preventive resiliency measures have been poorly discussed in the literature. Thus, this study investigated 
a multi-objective multi-echelon robust and RESCND problem under uncertainty and risks of disturbance. The OFs included minimizing the TC, TEI, and TNRN associated with the concerned SC. Besides, the issue of multiple OFs was countered by applying an augmented $\varepsilon$-constraint method. It was assumed that SC faces multiple disturbances so that facilities and transportation links would be partially and wholly disrupted, respectively. Furthermore, some preventive and contingency resilience strategies, including structural and network measures, were considered simultaneously to mitigate the detrimental consequences of disruptions. Structural resilience measures include MS, taking account into LT, HPEI by fortified facilities, and providing the ability to purchase from EI. Besides, the network non-resiliency measures consisted of NC, NCr, and FC. Ultimately, a Mulvey RO method was adopted to tackle usual uncertainties.

The SC consisted of six levels: primary and backup suppliers, warehouses, production centers, distribution centers, and first markets. Strategic decisions associated with the model included locating warehouses, production centers, and distribution centers; selecting proper backup suppliers; and determining the appropriate level for the FF. Besides, operational decisions were made in the incidence of disturbance and operational risks. They involved specifying the amount of product transported among facilities, the level of typical inventory kept at specific facilities, the amount of pre-positioned EI that had to be held, and the lost sale in the first markets. The OFs included minimizing the TC and the TNRN associated with the investigated SC simultaneously. The proposed model was implemented based on three datasets related to a case study of the steel industry in Iran to confirm the formulation's validation, effectiveness, and performance.

Numerical results indicated that MS, LT, and FF as resilience strategies would bring about the greatest costefficiency in the case study. Moreover, observations revealed that the fortified SC would be highly economically viable in the long run due to the reduction of costs resulting from lost sales, unnecessary inventory holding, and the company's credit risk. Managerial results confirmed that a resilient SC of the steel industry would provide the raw materials required by the production centers with the highest quality and in the shortest possible time through cooperation with reliable primary and backup suppliers. Moreover, due to the possibility of LT of end products between production centers during unforeseen disturbances, demands from customers and parent industries for steel alloys would be met. Accordingly, considering operational risk and disruptions simultaneously in the SC increased the costs; nonetheless, it would be worthwhile in the long run. Besides, a resilient system could prevent vulnerabilities using preventive and mitigation strategies and improve capabilities to mitigate the damages of the risks and decrease the costs.

Regardless of its contributions, our study had some limitations that can be considered as avenues for further research. As the investigated SC was exposed to numerous disturbances, employing some more precautionary and contingency strategies with proven successes can enhance the resilience capabilities of the system during disruptions. Besides, some resilience measures such as FF, considering excess capacity, decreasing the ND, and providing backup features can be applied to the proposed model to promote the SC's operational readiness and vulnerability tolerance during disturbances. Regarding the significance of SC sustainability in the global competitive market, in addition to economic issues, environmental and social dimensions associated with SC strategic and operational decision-making must be considered. Moreover, future studies can consider a scheduling or routing sub-problem to achieve the minimum total travel time to mitigate environmental impacts. Due to the significance of customer satisfaction in the business environment, meeting customer demands by considering backlog shortages is crucial in the SC and can improve demand fulfillment. Given the dimensions of the problem studied, an efficient and exact solution algorithm such as Lagrangian relaxation, Benders decomposition, and cut and column or various metaheuristic mechanisms should be adopted to reduce the execution time. 


\begin{tabular}{ll}
\hline List of Abbreviations \\
\hline SC & Supply Chain \\
SCM & Supply Chain Management \\
RESCND & Resilient Supply Chain Network Design \\
SCND & Supply Chain Network Design \\
SCRES & Supply Chain Resilience \\
TC & Total Cost \\
TEI & Total Environmental Impact \\
TSSP & Two-Stage Stochastic Programming \\
RO & Robust Optimization \\
TNRN & Total Non-Resiliency of the Network \\
NC & Node Complexity \\
FC & Flow Complexity \\
FCr & Flow Criticality \\
NCr & Node Criticality \\
ND & Network Density \\
EI & Emergency Inventory \\
LT & Lateral Transshipment \\
OF & Objective Function \\
SBR & Scenario-Based Robust \\
SR & Solution Robustness \\
MR & Model Robustness \\
VW & Variability Weight \\
RAW & Risk Aversion Weight \\
DUF & Demand Under-fulfillment \\
EV & Expected Value \\
FF & Fortification of Facilities \\
MS & Multiple Sourcing \\
HPEI & Holding Pre-positioned Emergency Inventory \\
PBF & Providing Backup Facilities \\
\hline &
\end{tabular}

\section{Appendix A.}

Research Highlights are proposed as follows:

- Improving economic, environmental and resilience performance of the supply chain simultaneously.

- Developing enhanced disruption management by considering structural resilience strategies and network non-resiliency measures, simultaneously.

- Applying hybrid precautionary and contingency strategies to fortify an organization's structure and network.

- Improving real-world conformity by taking into account multiple disruptions in facilities and routes among them.

- Tackling usual operational risks associated with the turbulent business environment by employing proper robust optimization technique.

The abstract has been graphically summarized as follows: 


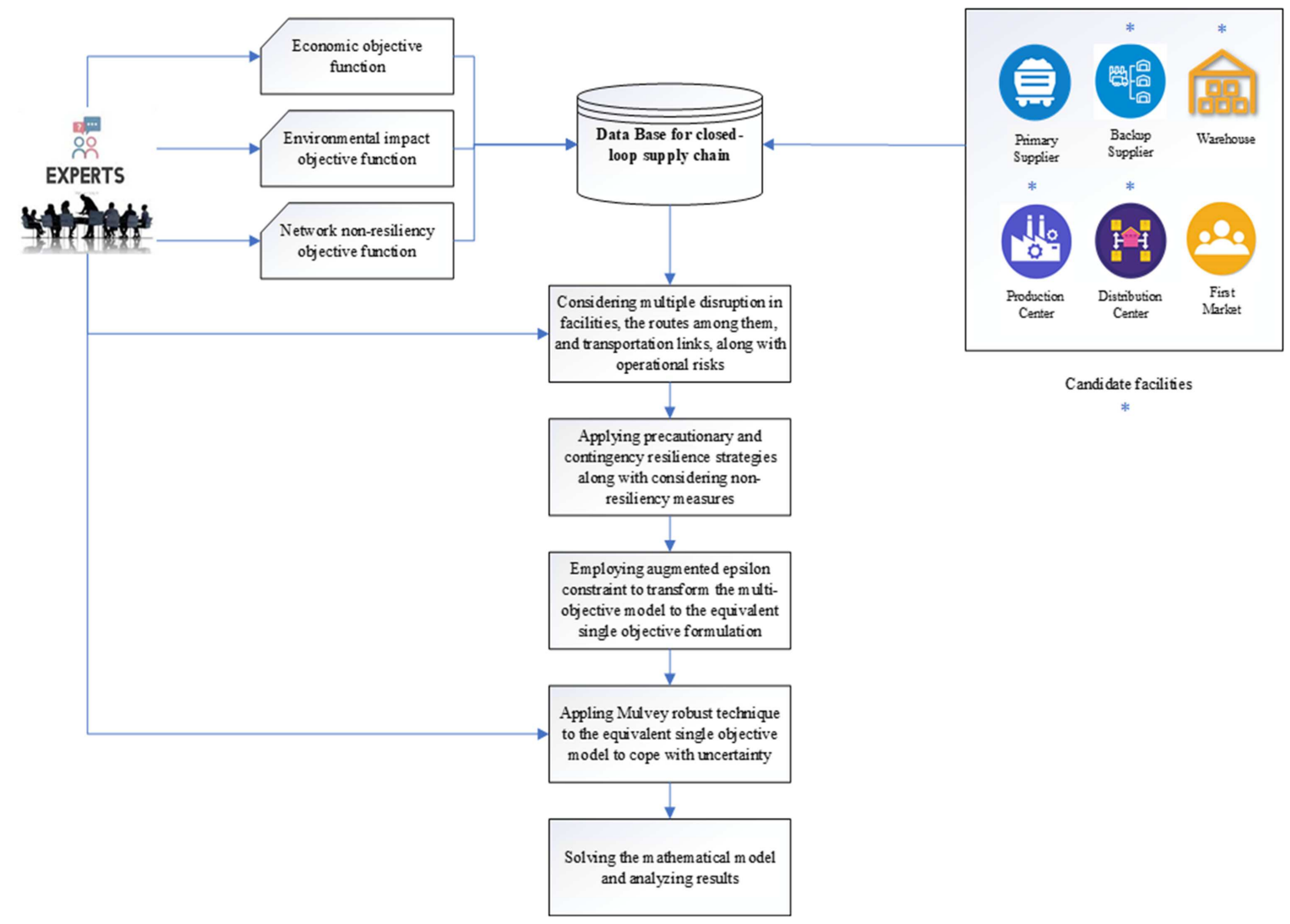

Acknowledgements. The authors are grateful to Prof Nelson Maculan and two anonymous reviewers of this journal for their helpful comments and suggestions for improving the paper.

\section{REFERENCES}

[1] N. Azad, G.K. Saharidis, H. Davoudpour, H. Malekly and S.A. Yektamaram, Strategies for protecting supply chain networks against facility and transportation disruptions: an improved Benders decomposition approach. Ann. Oper. Res. 210 (2013) $125-163$.

[2] D. Bertsimas and M. Sim, The price of robustness. Oper. Res. 52 (2004) 35-53.

[3] D. Bertsimas, V. Gupta and N. Kallus, Data-driven robust optimization. Math. Program. 167 (2018) $235-292$.

[4] A. Bhattacharya, J. Geraghty, P. Young and P. Byrne, Design of a resilient shock absorber for disrupted supply chain networks: a shock-dampening fortification framework for mitigating excursion events. Prod. Plan. Control. 24 (2013) $721-742$.

[5] S. Chopra and M. Sodhi, Supply-chain breakdown. MIT Sloan Manage. Rev. 46 (2004) 53-61.

[6] E. Dehghani, M.S. Jabalameli, A. Jabbarzadeh, M.S.J.C. Pishvaee and C. Engineering, Resilient solar photovoltaic supply chain network design under business-as-usual and hazard uncertainties. Comput. Chem. Eng. 111 (2018) $288-310$.

[7] E. Dehghani, M.S. Jabalameli and A. Jabbarzadeh, Robust design and optimization of solar photovoltaic supply chain in an uncertain environment. Energy 142 (2018) 139-156.

[8] M.H. Dehghani Sadrabadi, R. Ghousi and A. Makui, An enhanced robust possibilistic programming approach for forward distribution network design with the aim of establishing social justice: a real-world application. J. Ind. Syst. Eng. 12 (2019) $76-106$.

[9] M.H. Dehghani Sadrabadi, A. Jafari Nodoushan and A. Bozorgi-Amiri, Resilient supply chain under risks: a network and structural perspective. Iran. J. Manage. Stud. (2020). DOI: 10.22059/ijms.2020.306292.674139.

[10] M. Eskandarpour, P. Dejax and O. Péton, Multi-directional local search for sustainable supply chain network design. Int. J. Prod. Res. 59 (2021) 412-428.

[11] M. Falasca, C.W. Zobel and D. Cook, A decision support framework to assess supply chain resilience. In: Proceedings of the 5th International ISCRAM Conference (2008). 
[12] M. Fattahi, K. Govindan and E. Keyvanshokooh, Responsive and resilient supply chain network design under operational and disruption risks with delivery lead-time sensitive customers. Transp. Res. Part E: Logistics Transp. Rev. 101 (2017) 176-200.

[13] P. Garcia-Herreros, J.M. Wassick and I.E. Grossmann, Design of resilient supply chains with risk of facility disruptions. Ind. Eng. Chem. Res. 53 (2014) 17240-17251.

[14] A. Ghavamifar, A. Makui and A.A. Taleizadeh, Designing a resilient competitive supply chain network under disruption risks: a real-world application. Transp. Res. Part E: Logistics Transp. Rev. 115 (2018) 87-109.

[15] N. Gunantara, A review of multi-objective optimization: methods and its applications. Cogent Eng. 5 (2018) 1502242.

[16] M. Hajiaghaei-Keshteli and A.M.F. Fard, Sustainable closed-loop supply chain network design with discount supposition. Neural Comput. App. 31 (2019) 5343-5377.

[17] B. Hamdan and A. Diabat, Robust design of blood supply chains under risk of disruptions using Lagrangian relaxation. Transp. Res. Part E: Logistics Transp. Rev. 134 (2020) 101764.

[18] S.-M. Hosseini-Motlagh, M.R.G. Samani and F.A. Saadi, A novel hybrid approach for synchronized development of sustainability and resiliency in the wheat network. Comput. Electron. Agric. 168 (2020) 105095.

[19] A. Jabbarzadeh, B. Fahimnia and S. Seuring, Dynamic supply chain network design for the supply of blood in disasters: a robust model with real world application. Transp. Res. Part E: Logistics Transp. Rev. 70 (2014) 225-244.

[20] A. Jabbarzadeh, B. Fahimnia and F. Sabouhi, Resilient and sustainable supply chain design: sustainability analysis under disruption risks. Int. J. Prod. Res. 56 (2018) 5945-5968.

[21] S.C. Leung, S.O. Tsang, W.-L. Ng and Y. Wu, A robust optimization model for multi-site production planning problem in an uncertain environment. Eur. J. Oper. Res. 181 (2007) 224-238.

[22] J.T. Margolis, K.M. Sullivan, S.J. Mason and M. Magagnotti, A multi-objective optimization model for designing resilient supply chain networks. Int. J. Prod. Econ. 204 (2018) 174-185.

[23] G. Mavrotas, Effective implementation of the $\varepsilon$-constraint method in multi-objective mathematical programming problems. Appl. Math. Comput. 213 (2009) 455-465.

[24] A. Mohammed, I. Harris, A. Soroka and R. Nujoom, A hybrid MCDM-fuzzy multi-objective programming approach for a G-Resilient supply chain network design. Comput. Ind. Eng. 127 (2019) 297-312.

[25] J.M. Mulvey, R.J. Vanderbei and S.A. Zenios, Robust optimization of large-scale systems. Oper. Res. 43 (1995) $264-281$.

[26] A. Nikas, A. Fountoulakis, A. Forouli and H. Doukas, A robust augmented $\varepsilon$-constraint method (AUGMECON-R) for finding exact solutions of multi-objective linear programming problems. Oper. Res. (2020) 1-42. DOI: 10.1007/s12351-020-00574-6.

[27] M. Nili, S.M. Seyedhosseini, M.S. Jabalameli and E. Dehghani, A multi-objective optimization model to sustainable closed-loop solar photovoltaic supply chain network design: a case study in Iran. Renew. Sustainable Energy Rev. 150 (2021) 111428.

[28] M. Nili, S.M. Seyedhosseini, M.S. Jabalameli and E. Dehghani, An integrated model for designing a bi-objective closed-loop solar photovoltaic supply chain network considering environmental impacts: a case study in Iran. J. Ind. Syst. Eng. 13 (2021) 243-280.

[29] S.V. Nooraie and M.M. Parast, Mitigating supply chain disruptions through the assessment of trade-offs among risks, costs and investments in capabilities. Int. J. Prod. Econ. 171 (2016) 8-21.

[30] E. Olivares-Benitez, R.Z. Ríos-Mercado and J.L. González-Velarde, A metaheuristic algorithm to solve the selection of transportation channels in supply chain design. Int. J. Prod. Econ. 145 (2013) 161-172.

[31] A. Pavlov, D. Ivanov, D. Pavlov and A. Slinko, Optimization of network redundancy and contingency planning in sustainable and resilient supply chain resource management under conditions of structural dynamics. Ann. Oper. Res. (2019) 1-30. DOI: $10.1007 / \mathrm{s} 10479-019-03182-6$.

[32] T.J. Pettit, J. Fiksel and K.L. Croxton, Ensuring supply chain resilience: development of a conceptual framework. J. Bus. Logistics 31 (2010) 1-21.

[33] L. Purvis, S. Spall, M. Naim and V. Spiegler, Developing a resilient supply chain strategy during "boom" and "bust". Prod. Plan. Control 27 (2016) 579-590.

[34] S. Radhakrishnan, B. Harris and S. Kamarthi, Supply chain resiliency: a review. In: Supply Chain Risk Management. Springer (2018) 215-235.

[35] S. Rezapour, R.Z. Farahani and M. Pourakbar, Resilient supply chain network design under competition: a case study. Eur. J. Oper. Res. 259 (2017) 1017-1035.

[36] F. Sabouhi and M.S. Jabalameli, A stochastic bi-objective multi-product programming model to supply chain network design under disruption risks. J. Ind. Syst. Eng. 12 (2019) 196-209.

[37] F. Sabouhi, M.S. Pishvaee and M.S. Jabalameli, Resilient supply chain design under operational and disruption risks considering quantity discount: a case study of pharmaceutical supply chain. Comput. Ind. Eng. 126 (2018) 657-672.

[38] F. Sabouhi, M.S. Jabalameli, A. Jabbarzadeh and B. Fahimnia, A multi-cut L-shaped method for resilient and responsive supply chain network design. Int. J. Prod. Res. 58 (2020) 7353-7381.

[39] T. Sawik, Selection of resilient supply portfolio under disruption risks. Omega 41 (2013) 259-269.

[40] Y. Sheffi and J.B. Rice Jr, A supply chain view of the resilient enterprise. MIT Sloan Manage. Rev. 47 (2005) 41.

[41] D. Shi, A review of enterprise supply chain risk management. J. Syst. Sci. Syst. Eng. 13 (2004) 219-244.

[42] L. Silbermayr and S. Minner, Dual sourcing under disruption risk and cost improvement through learning. Eur. J. Oper. Res. 250 (2016) 226-238.

[43] R. Sreedevi and H. Saranga, Uncertainty and supply chain risk: the moderating role of supply chain flexibility in risk mitigation. Int. J. Prod. Econ. 193 (2017) 332-342. 
[44] S. Torabi, M. Baghersad and S. Mansouri, Resilient supplier selection and order allocation under operational and disruption risks. Transp. Res. Part E: Logistics Transp. Rev. 79 (2015) 22-48.

[45] S.A. Torabi, R. Giahi and N. Sahebjamnia, An enhanced risk assessment framework for business continuity management systems. Saf. Sci. 89 (2016) 201-218.

[46] P. Vaez, F. Sabouhi and M.S. Jabalameli, Sustainability in a lot-sizing and scheduling problem with delivery time window and sequence-dependent setup cost consideration. Sustainable Cities Soc. 51 (2019) 101718.

[47] P. Vaez, A. Jabbarzadeh and N. Azad, Designing a scheduling decision support system for the skin pass line: a case study of the steel finishing line. Proc. Inst. Mech. Eng. Part B: J. Eng. Manuf. 234 (2020) 1640-1655.

[48] B. Zahiri, J. Zhuang and M. Mohammadi, Toward an integrated sustainable-resilient supply chain: a pharmaceutical case study. Transp. Res. Part E: Logistics Transp. Rev. 103 (2017) 109-142.

[49] M. Zhalechian, S.A. Torabi and M. Mohammadi, Hub-and-spoke network design under operational and disruption risks. Transp. Res. Part E: Logistics Transp. Rev. 109 (2018) 20-43.

[50] J. Zhao and G.Y. Ke, Optimizing emergency logistics for the offsite hazardous waste management. J. Syst. Sci. Syst. Eng. 28 (2019) 747-765.

[51] L. Zhen, D. Zhuge and J. Lei, Supply chain optimization in context of production flow network. J. Syst. Sci. Syst. Eng. 25 (2016) 351-369.

\section{Subscribe to Open (S20) A fair and sustainable open access model}

This journal is currently published in open access under a Subscribe-to-Open model (S2O). S2O is a transformative model that aims to move subscription journals to open access. Open access is the free, immediate, online availability of research articles combined with the rights to use these articles fully in the digital environment. We are thankful to our subscribers and sponsors for making it possible to publish this journal in open access, free of charge for authors.

\section{Please help to maintain this journal in open access!}

Check that your library subscribes to the journal, or make a personal donation to the $\mathrm{S} 2 \mathrm{O}$ programme, by contacting subscribers@edpsciences.org

More information, including a list of sponsors and a financial transparency report, available at: https://www. edpsciences.org/en/maths-s2o-programme 